\title{
Estimation of the Present Status of the Species \\ Based on the Theoretical Bounds of Environmental Noise Intensity: An Illustration Through a Big Abundance Data and Simulation
}

\author{
Ayan Paul \\ Indian Statistical Institute \\ Nabakumar Ghosh \\ Acharya Jagadish Chandra Bose College \\ Sabyasachi Bhattacharya ( $\sim$ sabyabhatta@gmail.com ) \\ Indian Statistical Institute https://orcid.org/0000-0002-6432-6988
}

\section{Research Article}

Keywords: Bifurcation, Chaos, Theta-logistic model, GPDD, Probability of extinction, Stochasticity

Posted Date: December 16th, 2021

DOI: https://doi.org/10.21203/rs.3.rs-1072962/v1

License: (c) (1) This work is licensed under a Creative Commons Attribution 4.0 International License.

Read Full License 
Estimation of the present status of the species based on the theoretical bounds of environmental noise intensity: An illustration through a big abundance data and simulation

\footnotetext{
Ayan Paul $^{a}$, Nabakumar Ghosh ${ }^{b}$, Sabyasachi Bhattacharya ${ }^{a *}$

a Agricultural and Ecological Research Unit, Indian Statistical Institute, 203, B. T. Road, Kolkata, 700108, India.

${ }^{b}$ Department of Mathematics, Acharya Jagadish Chandra Bose College, 1/1B, AJC Bose Rd, Kolkata, 700020, India.
}

\footnotetext{
${ }^{*}$ Corresponding author

Email addresses: ayaninspire@gmail.com $\left(\right.$ Ayan Paul $\left.{ }^{a}\right)$, nkghosh37@gmail.com (Nabakumar Ghosh ${ }^{b}$, sabyabhatta@gmail.com (Sabyasachi Bhattacharya ${ }^{a}$ )
} 


\section{Abstract}

Sibly et al. (2005) described that most species have a fundamental characteristic to follow the thetalogistic growth trait with the convex downward trend. The fundamental yardstick of this research work builds under the deterministic setup, whereas the involvement of the external noise in any growth system is inevitable. But, the involvement of external affairs in any species growth can't be well judged only through its density dependence; it requires a further assessment. So, we frame the theta-logistic model with the stochastic analog in two directions, i.e., the discrete and continuous setup. The analysis of the discrete stochastic model is manifested by the bifurcation analysis, which shows that the attainment of the chaotic regime enhances with the increase in noise intensity level. Although the role of chaos in species extinction is debatable, a literature survey suggests that chaos with stochasticity accelerates the extinction of species. Similarly, in the case of the continuous version, we performed a theoretical study on the stochastic theta-logistic model to provide a critical value of the noise intensity parameter. This threshold magnitude act as the sustainability criteria of any species environmental tolerability. In this connection, we use the data of four major taxonomic groups, i.e., Bird, Insect, Mammal, and Fish, from the GPDD database and classify the species based on environmental sensitivity. The high sensitive species have a low tolerance level, associated with the small magnitude of environmental noise intensity parameter. Moreover, the simulation prediction model on these four taxonomic classes also shows that the overall extinction probability of the Bird is almost negligible for the current time window.

Keywords: Bifurcation, Chaos, Theta-logistic model, GPDD, Probability of extinction, Stochasticity.

\section{Introduction}

A fundamental question in population ecology concerns the relationship between the population size and its per capita growth rate (pgr). This relationship has vital implications for understanding the animal lives, sustainability, and risk status of the species. A seminal contribution by Sibly et al. (2005) established this relationship using density regulated population growth model based on the data of four taxonomic groups such as Birds, Insects, Mammals, and Bony fishes available in the Global Population Dynamics Database (GPDD) (NERC Centre for Population Biology, 2010). For some time series of species abundance in this database, the density-regulated parameters have been allowed to take negative values, which ultimately lead to a negative estimate of instantaneous growth rate, an unrealistic phenomenon of the population ecology. But, Bhowmick et al. (2015) addressed this issue and provided a settlement by proposing a more general model incorporating cooperation and competition.

Although Sibly et al. (2005)'s proposal of using theta logistic model for understanding the pgr-density relationship suffers from some mathematical and model-specific limitations, it provides some fundamental insights into the population dynamics. The study of Sibly et al. (2005) and a series of sequel studies (Reynolds and Freckleton, 2005; Bhowmick et al., 2015) reached the same conclusion that most of the pgr-density relationship is concave upward (convex) in nature except for large mammals. This phenomenon can be well described by the theta-logistic model having the density regulation parameter less than unity. This phenomenon is probably because many species would like to spend most of their lifespan much below the carrying capacity because of its better survival prospect and optimizing habitat rationing. We know that carrying capacity is the only stable equilibrium point, and so naturally, species would like to spend most of their life span around the carrying capacity. There is some evidence of nonmonotonic Allee type relationships between density and pgr, which is negligible in numbers compared to a concave upward relationship. Note that Sibly et al. (2005), and Bhowmick et al. (2015) found that the percentage of species following the Allee, cooperative growth mechanism is very much negligible in 
respect to the theta-logistic growth trait. The question persists whether the species are safe while adopting the theta-logistic pattern for their density-pgr relationship under the realistic stochastic environment.

The fundamental yardstick of Sibly et al. (2005)'s work builds under the deterministic setup, which can well describe through the first-order differential equation. It is worth mentioning that the theta-logistic model's discrete analog can well represent through the extended Ricker setup. A complete picture of the stable state can be obtained through the bifurcation diagram by varying the parameter instantaneous growth rate for fixed magnitudes of density regulated and carrying capacity parameters. Moreover, we can also have a clear concept of the estimated population size for which the species may enter sequentially into the limit cycle and chaotic dynamics.

Although it is a matter of debate whether the chaos is a precursor of extinction or not, Bhowmick et al. (2015) are informative in this connection. In a conclusive statement, Bhowmick et al. (2015) suggest that the probability of local extinction increases when the population enters into the regime of chaos. Ritchie (1992) demonstrates that if chaotic dynamics occur more often, the local population is almost inevitable. Thomas et al. (1980) pointed out that deterministic chaos is sometimes enhanced the probability of extinction. For example, nonlinear dynamics increase the likelihood of species local and global extinction in brine shrimp experimental populations. Huisman and Weissing (1999) describe that chaos is not always harmful, and the maintenance of chaos is desirable for reducing the probability of global extinction to maintain species biodiversity. But chaos has not always a detrimental effect on the ecosystem (Ruxton, 1994); instead, the system managers should regulate certain unwanted fluctuations (Bhowmick et al., 2015). They will have to be careful whether the population size declines the viable size or not. In thermodynamics, entropy measures the randomness of the environment. In nonlinear dynamics, chaos is the measure of this system's entropy (Waliszewski and Konarski, 2005). Hence, the beginning of chaotic dynamics may not always lead to extinction. Still, in the transition of chaotic dynamics, population growth is very irregular and unpredictable. Even a tiny external perturbation may lead the population to extinction, leaving a long-term impact on the population dynamics (Schreiber, 2003). Summarizing the above debates and cross debates, we can conclude that chaos with environmental stochasticity increases extinction probability (Berryman and Millstein, 1989). Still, the coupled population can avoid global extinction utilizing chaotic dynamics (Allen et al., 1993).

Lande (1993) point out that the incorporation of stochasticity will profoundly impact species growth as most of the population dynamics drives by external fluctuations. IGR is the most vital parameter, which has been affected by demographic stochasticity (Loeschcke and Seitz, 1991). There are also many instances where IGR is affected by both environmental and demographic stochasticity (Méndez et al., 2010). Mathematically, suppose we incorporate stochasticity in IGR of the theta-logistic model by introducing the Wiener process. In that case, the growth equations take the form of a stochastic differential equation with the multiplicative noise structure.

A key question for the population biologists is whether there is any theoretical bound for quantifying the intensity of stochastic fluctuation based on the theta-logistic model's parameters. The selection of the theta-logistic model is for its application for explaining the relationship of pgr-density for most of the species. The empirical estimate of this bound based on the real data will help provide information on the actual status of the species sustainability and extinction. A species will be threatened due to the multiplicative noise variation, although; they exhibit stable dynamics under deterministic theta logistic setup. In this paper, we have three folds objectives: (i) Estimate the theoretical bounds of 
the theta-logistic model by incorporating the multiplicative noise through IGR, (ii) comment on the species sustainability and risk status by evaluating the empirical estimate of the above bounds based on the GPDD data sets, (iii) the abundance data for species of several taxonomic groups are available in different past time windows. Based on this historical data, we like to predict the species present status through the probability of extinction.

\section{Aim of the present study}

\section{Non-overlapping vs. overlapping generations: Discrete and continuous system}

Species growth traits are well explained through the theta-logistic model (Sibly et al., 2005). Most of the natural systems are governed by external noises. A key question of ecology is how much noise intensity a system can tolerate to preserve the species sustainability. So, it is clear that the noisy stochastic system may not be stable, although the deterministic trait can reach a steady state. The species growth regulation can be viewed in two ways. Continuous setup is appropriate for the species with over-lapping generation. Most interestingly, some species have life cycles maintained through non-overlapping generations (Rana et al., 2014). In this case, the discrete analog of the theta-logistic model is functional. Another critical question will arise how an experimental biologist can understand the extinction status of some species under a discrete growth rate. The advantage of a discrete system is that a single species model can exhibit the different dynamical phases such as stable, limit cycle, and chaos under varying parameters. We need at least a two-dimensional system to observe the chaos in a continuous setup (Mortoja et al., 2020).

\section{Does chaos act as an onset of species extinction?}

It is debatable that whether chaos can be viewed as a precursor of extinction. Chaos is a non-repeating, erratic fluctuation trajectory that is bounded and sensitive to the initial condition. The system managers will have to be careful whether the population size declines the viable population size or not. Population size below this critical threshold may enhance the risk of extinction if stochasticity is present in the system. If we consider any simple environment, small vegetation-eating animals with high reproductive rates eat almost all the vegetation in one year. The following year, the vegetation will not have recovered, but the species richness will still be very high. Thus the high growth rate causes a disconnect between the actual population size and the adverse effects of those individuals comprising the population. The negative effects of the actions of individuals (e.g., resource consumption) are felt by the offspring of those individuals rather than the individuals themselves. Hence, the birth of chaos occurs in the ecosystem. However, chaos is not always detrimental. Systematic chaotic intervention is desirable for reducing the probability of global extinction (Huisman and Weissing, 1999). Note that a bifurcation diagram is a formidable tool for understanding the chaotic dynamics.

\section{Lyapunov exponent as an indicator of chaos}

Several authors (Berryman and Millstein, 1989; Thunberg, 2001; Dennis et al., 2003) discuss that Lyapunov exponent (henceforth, LE) should illustrate the chaotic behavior of any species be a formidable measure. The positivity of the LE signifies the chaotic regime into the dynamical system (see the diagram ref Deterministic discrete theta-logistic bifurcation diagram). Berryman and Millstein (1989) state that this positivity depends on two factors. The factors are that the production of the offspring should be regular. Positive feedback effects dominate that dynamical system. Moreover, species richness is displaced far from their equilibrium points due to the negative overcompensating effect. In that situation, 
positive feedback behavior must dominate.

\section{Chaos in discrete theta-logistic model: an illustration}

One of the primary objectives of the present study is to predict the species extinction status and estimate the amount of environmental perturbation a species can tolerate. Most of the species under consideration follow the law of overlapping generations. So, we focused our study on the continuous system for identifying the species status and the theoretical bound of environmental fluctuations. Before exploring the continuous version of the theta-logistic model, we feel that it would be appropriate to monitor the discrete system through bifurcation analysis. We need to identify when the chaotic regime starts by varying the values of the reproduction parameter. A bifurcation diagram can determine parameter values for which the dynamical system moves to period-doubling, limit cycle, and extinction corridor. This analysis will be helpful to predict the local extinction status of the species. We focus our study on a non-overlapping species, Oncorhyncus gorbusha. We compare the estimated values of the reproduction parameter with its theoretical values for which the system will move to a chaotic regime. In order to understand the nature of the bifurcation diagram, one should follow the protocol of Thunberg (2001). In the spirit of Thunberg (2001), we propose a theorem to justify whether the diagram for the species possesses the one-humped character or not. The area of the hump is an indicator of the onset of the chaotic region: more the area, less the risk of entering the species into the chaotic zone.

\section{Species extinction status under continuous stochastic theta-logistic model}

We will express the theta-logistic growth model as a stochastic differential equation by incorporating the multiplicative noise in the reproduction term. We can theoretically estimate the upper bound of the environmental fluctuation using Ito calculus and the Lyapunov function. The risk status of the species can be identified by comparing the theoretical estimates with that of the fitted model estimate of the noise intensity parameter. This comparison is made based on a systematic and thorough study on 1780 time series of species from different taxonomic groups. A natural and fundamental question will automatically arise: What is the current status of the species concerning the calendar year 2021? We have planned to propose a simulation-based analysis for identifying the extinction status of the species, which is contemporary to the PVA analysis in IUCN.

\section{Theta-logistic model and density regulation}

The growth dynamics of any species is characterized by the convolution of two opposite growth pulses, i.e., (1) natural inherent affinity of the species for unlimited growth and (2) the nature's interference as the negative feedback. The overcompensatory dynamics of the density-dependent phenomena is one of the significant components behind these two characteristics (Nicholson, 1954; Kot, 2001; Bhowmick et al., 2015). The negative feedback rule of any ecosystem linger the species unlimited growth as a consequence based on the available resources and favorable conditions species have a chance to change their habitat through the adaptation on the stage of evolutionary theater due to the long time survival prospect (Johnson and Agrawal, 2003). Although several controversial statement exists (Andrewartha and Birch, 1984; Foley, 1994) regarding the influence of the density dependence, recently many authors (Fagan et al., 2001; Henle et al., 2004; Heering Jr and Reed, 2005; Lande et al., 2009) acknowledge the importance of it upon the species growth regulation. 
The classical model describing the density-dependent phenomena is the logistic growth law. The linear decrement of the relative growth rate with increasing population abundance will put a restriction on the explanation of density dependence through the logistic growth law (Ross, 2009). The study of Sibly et al. (2005) reveals that the density dependence is not well captured through the linear association between the species fitness and its abundance. The author demonstrates this relevance by showing that the fitness profile of most of the species follows a non-linear trend, i.e., either convex or concave. Several authors (Lande et al., 2009; Ross, 2009) also admit the non-linear associations between the species fitness and its abundance to illustrate the density dependence issue. In this connection, Sibly et al. (2005) uses the theta-logistic growth curve model to explain the non-linear trend in the species growth profile. The mathematical expression of this growth equation is given by

$$
\frac{d N(t)}{d t}=r N(t)\left(1-\left(\frac{N(t)}{K}\right)^{\theta}\right) .
$$

Here $N(t)$ represents the population density at any time $t$. The model parameters $K, \theta$ represent the carrying capacity and the curvature parameter of the theta-logistic model. Sibly et al. (2005) analyze the size-rgr relationship for 1780 time series from the Global Population Dynamics Database (GPDD) with this theta-logistic growth trait. The time series consists of four taxonomic groups, i.e., Aves, Insect, Fish, and Mammals. The author found that the density regulatory behavior in most species growth profiles is maintained by $\theta<1$. But, for the large mammals, this density regulation is followed by the relation $\theta>1$. Hence, it is clear that the theta-logistic growth trait is one of the stable dynamics in the species growth regulation. Apart from this growth regulation, some species follow the Allee growth law in their life cycle. Note that, Sibly et al. (2005) estimated this minor percentage as 0.2, and Bhowmick et al. (2015) evaluated this figure to be $3.92 \%$ for cooperative species, where cooperation is a particular case of an Allee effect.

\section{Discrete analog of theta-logistic model}

We have already mentioned that the theta-logistic growth dynamics well explain the size-rgr relationship in most of the species. Sibly et al. (2005) use the deterministic continuous set up to elucidate this scenario. It is worthy of mentioning that this continuous deterministic form of the theta-logistic law does not relate to species extinction. But, the one-dimensional discrete ricker map of the theta-logistic growth equation possesses several characteristics, viz., 2-period cycle, 3-period cycle, period doubling, limit cycle, chaos, etc., for a specific range of parameter value. Although the association of the chaotic dynamics with species extinction is still debatable, we believe that these unpredictable, chaotic dynamics certainly increase the probability of extinction very much. So, there is an urge to project a spotlight on the discrete dynamics of the theta-logistic growth law. The discretized version of theta-logistic law is given by

$$
N(t+1)=N(t)+r N(t)\left(1-\left(\frac{N(t)}{K}\right)^{\theta}\right),
$$

with the notations have their usual meaning.

\subsection{Discrete system and chaotic regime}

Chaotic oscillations are generally observed after attaining the asymptotic size. Hence, the maximum population size or the carrying capacity plays the role of attractor in any ecosystem. Thus, from the mathematical perspective, one can say that carrying capacity creates a basin of attraction after which 


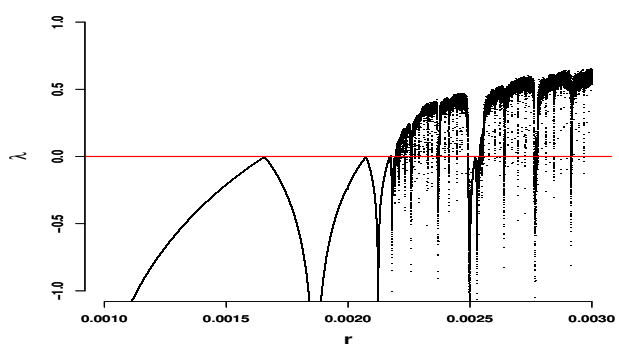

(a) $\theta=0.1$

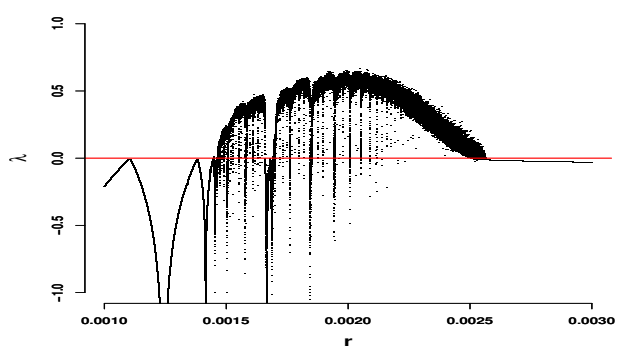

(c) $\theta=0.15$

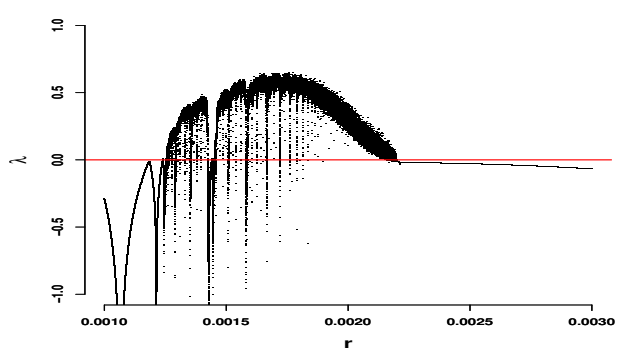

(e) $\theta=0.175$

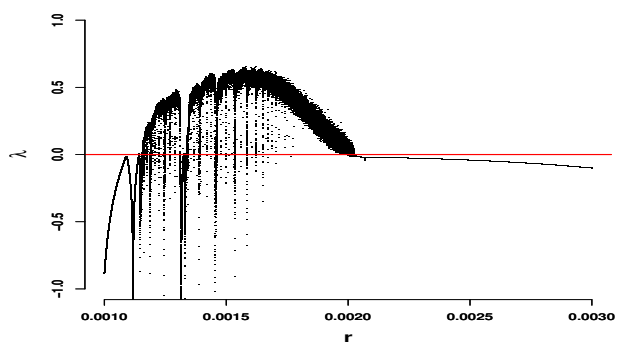

(g) $\theta=0.19$

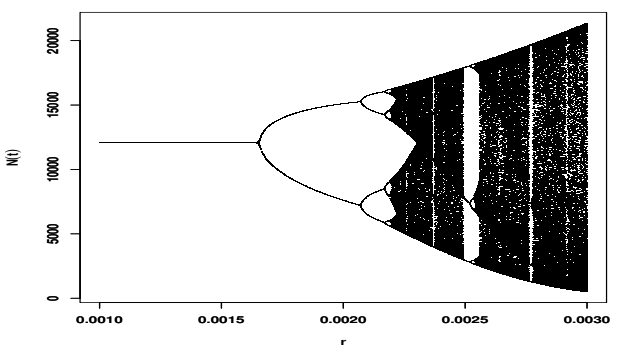

(b) $\theta=0.1$

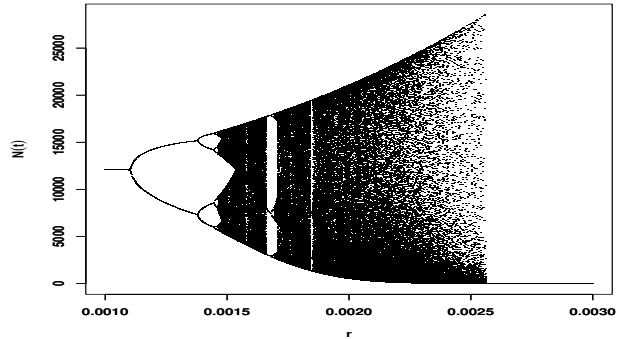

(d) $\theta=0.15$

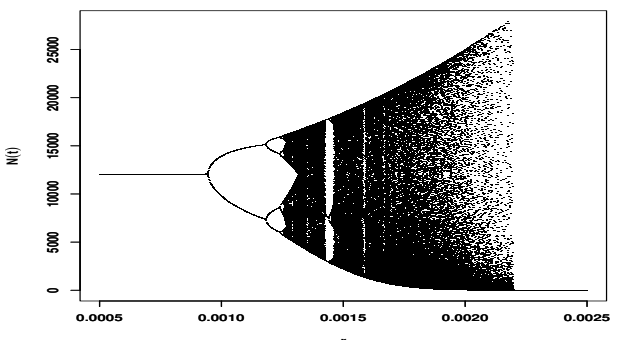

(f) $\theta=0.175$

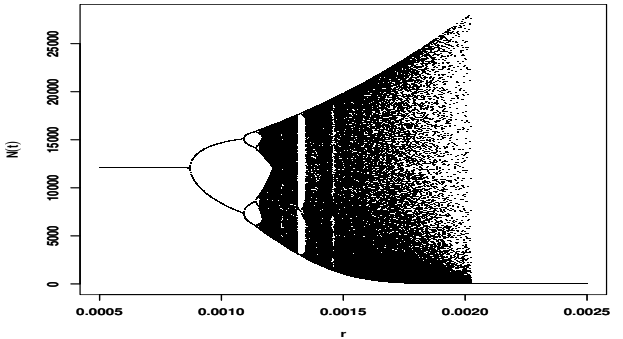

(h) $\theta=0.19$

Figure 1: Bifurcation diagram of the population size $(N(t))$ of Oncorhynchus gorbuscha (GPDD ID-1844) expressed by the deterministic growth equation 4.1 is present on the right panel of the figure. The left panel figures indicate the Lyapunov exponents. Here we use the estimated values of $K, \theta$ i.e., 12068.92, 0.1 respectively with the initial population size 6900 to draw this diagram. Note that in the sub-figures (c), (d); (e), (f); (g), (h) we gradually increase the magnitude of $\theta$ by $50 \%, 75 \%, 90 \%$ respectively to observe the dynamical change in the equilibrium points.

the orbital period of the growth trajectory becomes infinite. This would lead to chaos in the dynamic 
system. In this connection, we provide the following theorem to elucidate the analytical property of the bifurcation diagram.

Theorem 4.1. The bifurcation diagram of the discrete theta-logistic model 4.1 will be one-humped if $N(t+1)$ in equation 4.1 has the maximum at $N(t)=\left[\frac{(1+r) K^{\theta}}{r(1+\theta)}\right]^{\frac{1}{\theta}}$.

Proof. Let us consider $f(N(t), \theta)$ in the equation 4.1 by

$$
\begin{aligned}
& f(N(t), \theta)=N(t)+r N(t)\left(1-\left(\frac{N(t)}{K}\right)^{\theta}\right) \\
& \Longrightarrow f(N(t), \theta)=N(t)+r N(t)-\frac{r N^{\theta+1}(t)}{K^{\theta}} \\
& \Longrightarrow f^{\prime}(N(t), \theta)=1+r-\frac{(1+\theta) r N^{\theta}(t)}{K^{\theta}} \\
& \Longrightarrow f^{\prime \prime}(N(t), \theta)=-\frac{\theta(1+\theta) r N^{\theta-1}(t)}{K^{\theta}}<0
\end{aligned}
$$

In order to find the maximum point we need to solve the equation $f^{\prime}(N(t), \theta)=0$, which provides $N(t)=\left[\frac{(1+r) K^{\theta}}{r(1+\theta)}\right]^{\frac{1}{\theta}}$.

Note that, Thunberg (2001) proposed that any one dimensional map from an interval $I$ to itself will be uni-modal (One humped) if $\exists$ any point $c \in I=[a, b]$ such that $f(N(t), \Theta)$ has a unique maximum value at $c$, i.e., the function is monotonically increasing on $[a, c)$ and then decreasing on the interval $(c, b]$.

Here the one-dimensional map of the theta-logistic model 4.1 is confined in the closed interval $[0, K]$. Moreover, the specified result mentioned above depicts that $N(t) \in[0, K]$. This ensures that the bifurcation diagram should possess the one-humped characteristics.

The numerical output of the bifurcation diagram (see the right panel of the figure 1) also depicts that it possesses the one-humped characteristics. The stability analysis in difference equation (Kot, 2001) shows that the discrete theta logistic system 4.1 becomes stable if it maintains the condition $0<r \theta K<1$. In addition, the growth equation 4.1 undergoes damping oscillation for $1<r \theta K<2$, where the orbital period of the one-humped mapping 4.1 revolve within the power of 2 . The beginning of the limit cycle is observed in the system 4.1 when $r \theta K>2$, i.e., the one-dimensional mapping is now beginning to follow an odd order period. The phase of the limit cycle then gradually converts to a chaotic stage when the period of the orbit becomes three (Li and Yorke, 2004).

Since the bifurcation diagram is produced by varying the parameter values, it would become necessary to identify the threshold value of the parameter responsible for attaining the chaos. The Lyapunov exponent (LE) should be the formidable measure in this case. It is defined as

$$
\lambda_{N_{0}}=\lim _{n \rightarrow \infty} \frac{1}{n} \sum_{i=0}^{n-1} \ln \left(\left|\frac{d f}{d N}\left(N_{i}\right)\right|\right)
$$

where $N_{0}$ be the fixed point of the mapping $N(t+1)=f(N(t), \Theta)$. The positive magnitude of LE concerning the bifurcation parameter predicts the onset of chaos in any deterministic discrete system. The numerical analysis of the expression 4.3 gives that critical value of the parameter for the discrete theta-logistic map. The horizontal red line in the left panel of the figure 1 is used for the separation of the positive and negative zone of the LE, which also helps to recognize the threshold value of the IGR 
parameter to accomplish the chaos. This bifurcation analysis is being carried out on the abundance data of Oncorhyncus gorbuscha (GPDD ID-1844) available on the GPDD website. The diagram reflects that the pink salmon can achieve their stable state with a very low reproduction rate.

Moreover, the chaotic regime also occurs with a minimal level of IGR. But, the reproduction rate of the pink salmon is not too low at the Dean Channel of British Colombia, Canada, from which the data is collected (Armstrong et al., 2018). We believe the deterministic process is unable to highlight the true story beyond the species growth regulation. In this connection, we develop the following section to nurture the species growth regulation in any noisy interactive system.

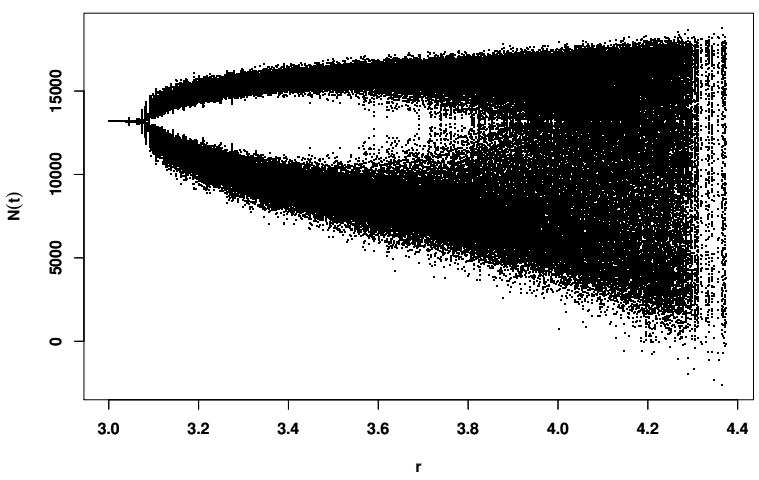

(a) $\sigma=3.29$

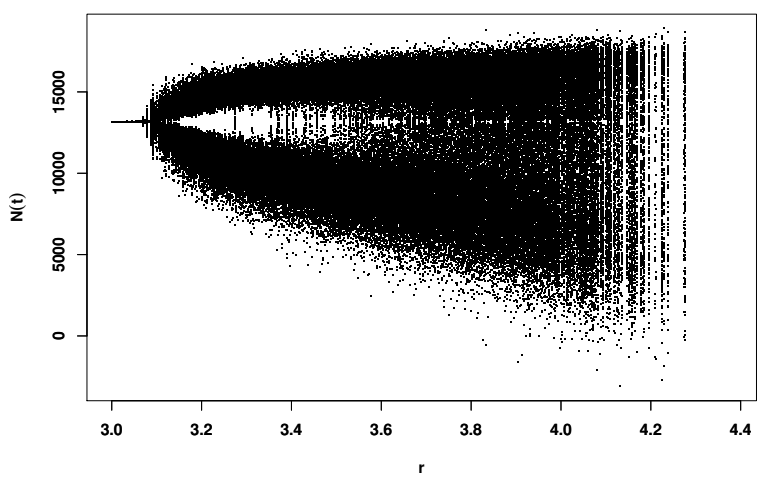

(c) $\sigma=5.7575$

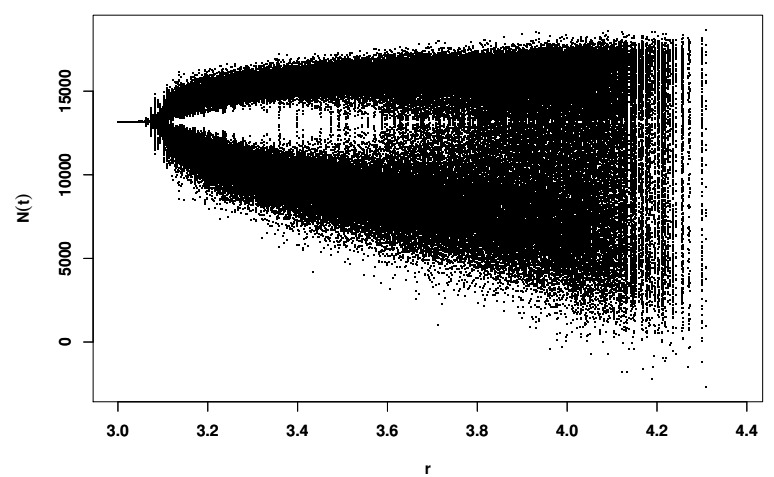

(b) $\sigma=4.935$

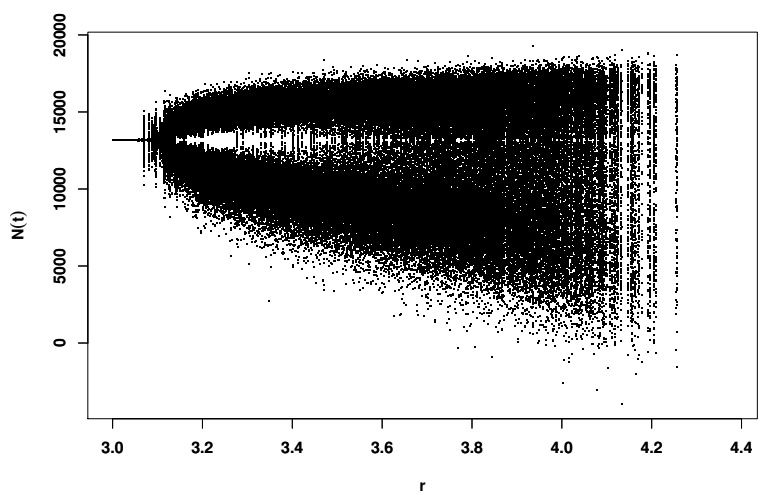

(d) $\sigma=6.251$

Figure 2: Bifurcation diagram of the population size $(N(t))$ of Oncorhynchus gorbuscha (GPDD ID-1844) expressed by the stochastic growth equation 5.3 with respect to the IGR $(r)$. Here we use the estimated values of $K, \theta$, $\sigma$, i.e., 13176.7, 0.65, 3.29 respectively with the initial population size 100 to draw this diagram. Note that in the sub-figures (b), (c), (d) we gradually increase the magnitude of $\sigma$ by $50 \%, 75 \%, 90 \%$ respectively to observe the dynamical change in the equilibrium points.

\section{Stochastic set up}

The growth dynamics of each species are affected by the internal and external disturbances (Chakraborty et al., 2017). The internal fluctuations among the species are very nuanced dynamics which, are well 
maintained through the density-dependent mechanism. But, the involvement of external affairs in any species growth can't be well judged only through its density dependence; it requires a further assessment. However, it is observed that in a large-sized population, fluctuations in the observed number of individuals are small. But, it seems that due to the small population size, internal fluctuation plays the role of a positive catalyst in the extinction of the species. In growth curve modeling, it is very much essential to incorporate the unexpected changes into the dynamical system (Chakraborty et al., 2017). Stochastic differential equations (SDE) is the natural choice to model any dynamical system when the species confront any randomness (Bishwal, 2008; Anderson, 2013). In addition, environmental fluctuation is a linchpin in any ecosystem. Therefore, most of the natural phenomena do not follow strictly deterministic laws rather oscillate randomly about some average value so that the deterministic equilibrium is no longer an absolutely fixed state (Arditi and Ginzburg, 1989). This analyzes the deterministic growth curve models under stochastic perturbations as a promising and important field in the present decade.

Any SDE can be formulated by introducing randomness into a deterministic dynamical system described by an ordinary differential equation (ODE). The simplest form of an SDE is given by

$$
d y(t)=G(y(t)) d t+\sigma d W(t)
$$

where $G(y(t))$ is a function representing the deterministic response of the system, $W(t)$ is a Wiener process representing the stochastic driving, and $\sigma$ is a scaling constant representing the intensity of the noise. The term $\sigma d W_{t}$ is the mathematical demonstration of the physical noise. Any external perturbations directly affect the species reproductive rate in such a way the parameter IGR $(r)$ will no longer be a constant one. As a consequence the parameter $r$ behaves like a random variable with certain mean $\bar{r}$ and standard deviation $\sigma \frac{d W(t)}{d t}$. This leads the population size of the species $(N(t))$ to a random variable. It is worthy of mentioning that the incorporation of such types of characteristics makes the deterministic theta-logistic model (3.1) the stochastic one, which the following SDE can explore.

$$
\frac{d N(t)}{d t}=\left(\bar{r}+\sigma \frac{d W(t)}{d t}\right) N(t)\left(1-\left(\frac{N(t)}{K}\right)^{\theta}\right) .
$$

Similarly in discrete set up the above equation 5.2 can be written as

$$
N(t+1)=N(t)+[\bar{r}+\sigma(W(t+1)-W(t))] N(t)\left(1-\left(\frac{N(t)}{K}\right)^{\theta}\right) .
$$

Remark 1. In a deterministic setup, there exist two equilibrium points for the theta-logistic model; one is the extinction state, i.e., 0 , and the other is near carrying capacity. Among these, 0 is the unstable equilibrium, and another is stable. But when we give a sufficient perturbation into the system, then the stability pattern changes. The zero stable equilibrium also acts as a stable one, and there may be a false interpretation. It can also be explained from the figure 3. The diagram exhibits that the simulated sample paths of Microtus arvalis (GPDD ID 9295) follow a to and fro movement in between the two fixed points, i.e., zero (0) and their asymptotic size. But the mean profile, i.e., the blue dashed line, preserves the deterministic sigmoidal property of underlying the growth dynamics.

It is worth mentioning that we use the bifurcation analysis for predicting the species growth status for the discrete setup. Moreover, for the continuous process, i.e., equation 5.2, we follow the standard protocol of the stochastic stability analysis. Now, for the sake of simplicity let us consider the transformation $y(t)=\left(\frac{N(t)}{K}\right)^{\theta}-1$ in the equation 3.1. Now, the modified version of 3.1 can be expressed as 


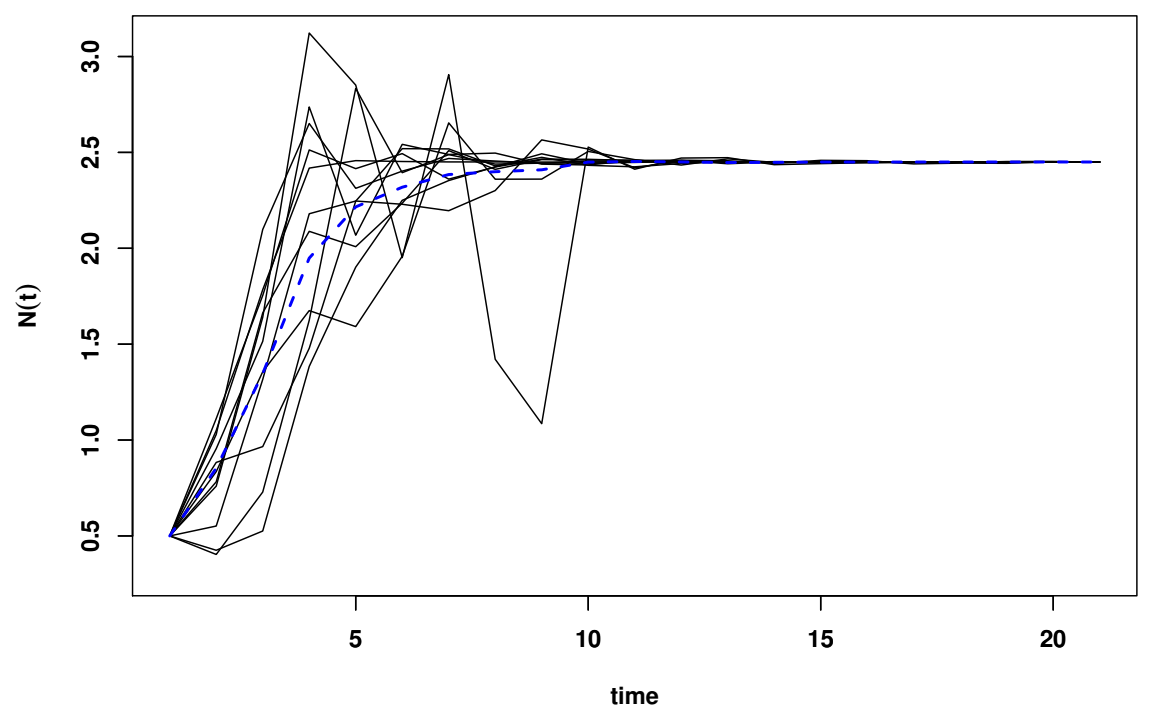

Figure 3: The simulated time series of the stochastic theta-logistic model (5.2) with $r=0.45, K=2.45, \theta=0.97, \sigma=1.43$. The solid (black) lines are the last $10 \%$ sample paths and the dashed (blue) line is the mean profile of all simulated sample paths. Note that the model parameters we have used here are being estimated from the growth data of Microtus arvalis (GPDD ID 9295) collected from the GPDD database.

$$
\begin{aligned}
& \frac{d}{d t}\left[K(1+y(t))^{\frac{1}{\theta}}\right]=r N(t)\left(1-\left(\frac{N(t)}{K}\right)^{\theta}\right)\left[\because y(t)=\left(\frac{N(t)}{K}\right)^{\theta}-1 \Longrightarrow N(t)=K(1+y(t))^{\frac{1}{\theta}}\right] \\
& \Longrightarrow \frac{d}{d t}\left[K\left(1+\frac{1}{\theta} y(t)\right)\right]=r N(t)\left(1-\left(\frac{N(t)}{K}\right)^{\theta}\right)[\because|y(t)|<1] \\
& \Longrightarrow \frac{K}{\theta} \frac{d y(t)}{d t}=r K(1+y(t))^{\frac{1}{\theta}}(-y(t)) \\
& \Longrightarrow \frac{d y(t)}{d t}=-r \theta K y(t)\left(1+\frac{1}{\theta} y(t)\right)
\end{aligned}
$$

Note that the substitution of $\theta=1$ in the equation 5.4 provide the same set-up for the logistic model (Golec and Sathananthan, 2003). So, in the spirit of Golec and Sathananthan (2003) we will perform our further analysis based on the equation (5.4). Note that the expression 5.4 is the linearized version of the original theta-logistic growth law. It is a well-known fact that the linearization technique helps to remove the non-linearity from any dynamical system (Saha et al., 2013; Bhowmick et al., 2016). We also follow this similar approach on the parameter $\theta$, which is responsible for the non-linearity in the theta-logistic model and thus obtain the equation (5.4).

Let us now suppose that growth rate IGR $(r)$ is a random variable with $r(t)=\bar{r}+\epsilon_{t}$, where $\bar{r}>0, \epsilon_{t}$ is a Gaussian white noise with a time varying intensity $\sigma^{2}$ such that $\epsilon_{t}=\sigma d W(t)$. So, the stochastic version of (5.4) can now be expressed by the following Ito-type initial value problem 


$$
d y(t)=-\bar{r} \theta K y(t)\left(1+\frac{1}{\theta} y(t)\right) d t-\theta \sigma K y(t)\left(1+\frac{1}{\theta} y(t)\right) d W(t)
$$

We consider the initial conditions $y_{0}>-1$, where needed, where $y(t)=y\left(t, y_{0}\right)$. The principle of convergence for $y(t)(\rightarrow 0)$ implies that $N(t) \rightarrow K$, while the convergence $y(t) \rightarrow-1$ implies $N(t) \rightarrow 0$. Thus the stability of zero solution of (5.4) will guarantee the stability of the equilibrium $N(t)=K$. The theorem (5.1) deals with the stochastic stability of the zero solution of the equation (5.5).

Theorem 5.1. Let us consider the stochastic $\theta$ logistic model 5.5. If

$$
\sigma^{2} \leq \frac{2 \bar{r}}{\theta K}
$$

holds for $t \geq 0$, the equilibrium position $y=0$ is stochastically stable i.e. for every $\epsilon \geq 0$ we have

$$
\lim P\left(\operatorname{Sup} \mid y\left(t, y_{0} \mid \geq \epsilon\right)=0,\right.
$$

when $y_{0} \rightarrow 0$.

If in addition,

$$
\bar{r}-\frac{1}{2} K \theta \sigma^{2} \geq 0
$$

for $t \geq 0$, the equilibrium position is stochastically asymptotically stable in large on the interval $(-\theta, \infty)$ in addition for every $y_{0} \geq-1$, we have

$$
P\left(\operatorname{limy}\left(t, y_{0}\right)=0\right)=1
$$

So the function (5.9) has an Ito differential

$$
d V(y(t))=\left[\frac{\partial v}{\partial t}+a \frac{\partial v}{\partial y(t)}+\frac{1}{2} b b^{T} \frac{\partial^{2} v}{\partial y^{2}(t)}\right]+b \frac{\partial v}{\partial y(t)} d W(t),
$$

where the first part denotes the Markov generator $(L V(t, y(t)))$ with $a$ and $b$ as the drift vector, diffusion matrix respectively.

Here,

$$
a=-\bar{r} \theta K y(t)\left(1+\frac{1}{\theta} y(t)\right)
$$

and

$$
b=\theta \sigma K y(t)\left(1+\frac{1}{\theta} y(t)\right)
$$

Thus,

$$
\begin{array}{r}
d V(y(t))=-K y^{2}(t)\left(\frac{\bar{r}}{\theta}-\frac{1}{2} \sigma^{2} K\right) d t-\frac{y^{2}(t) \sigma K}{\theta} d W(t) \\
\Longrightarrow \int_{s}^{t} d V(y(t))=\int_{t}^{s}-K y^{2}(t)\left(\frac{\bar{r}}{\theta}-\frac{1}{2} \sigma^{2} K\right) d t-\int_{s}^{t} \frac{y^{2}(t) \sigma K}{\theta} d W(t) \\
\Longrightarrow V(t, y(t))-V(s, y(s))=\int_{s}^{t} L V(t, y(t))-\int_{s}^{t} \frac{y^{2}(t) \sigma K}{\theta} d W(t)
\end{array}
$$


Here, $L V(t, y(t))=-K y^{2}(t)\left(\frac{\bar{r}}{\theta}-\frac{1}{2} \sigma^{2} K\right)$. This analytical form is indicated as the Markov generator. Now, for the sake of stochastic stability the Markov generator should be negative (Khasminskii, 2011). So, the required condition is given by,

$$
L V(t, y(t))=-K y^{2}\left(\frac{\bar{r}}{\theta}-\frac{1}{2} \sigma^{2} K\right) \leq 0 \Longrightarrow \sigma^{2} \leq \frac{2 \bar{r}}{\theta K} .
$$

Here the solution $y(t)=0$ i.e. a line contained in a domain $t \times U$. Moreover the Lyapunov function $V(t, y(t))$ is positive definite and $L V(t, y(t)) \leq 0$ for $y \neq 0$, then $y(t)=0$ be stable in probability, which is nothing but that the solution be stochastically stable, which proves the first part of the theorem. Moreover, $\inf _{t>0} V(t, y(t)) \rightarrow \infty$ as $|y(t)| \rightarrow \infty$. Hence, a sufficient condition for the solution $y(t)=0$ be stable in large is that $\exists$ a positive definite function $V(t, y(t))$ such that $L V(t, y(t))$ is negative definite and $\inf _{t>0} V(t, y(t)) \rightarrow \infty$ as $|y(t)| \rightarrow \infty$. This shows that the solution be asymptotically stochastically stable in large over the interval $(-\theta, \infty)$.

Remark 2. The model 5.5 reflects the impact of the density dependence with the stochastic analog in such a way that the ecologists can easily infer about the 'variation thresholds' beyond which the species have a chance of extinction. Based on the density-dependent selection process MacArthur (1962) conclude that the carrying capacity of any species is always maximized due to the principle of evolution. Although the conclusion made by MacArthur (1962) is acknowledged by Roughgarden (1979), Charlesworth et al. (1994) etc., recently Lande et al. (2009) shows that this proposition is not valid for the stochastic environment. The external fluctuations produce a trade-off between the selection of species with an increased intrinsic growth rate at the small abundance and the selection for larger carrying capacity at the high population density, summarizing the notion of the $r-K$ strategy (Pianka, 1970; Desharnais and Costantino, 1983; Boyce, 1984). The ubiquitous influence of the natural selection theory (' $r$ ' and ' $K$ ' selection strategy) on the species growth trait convey Fagan et al. (2001) to categorize the population dynamics into several groups. The association between the environmental noise intensity $(\sigma)$ and the intrinsic growth rate $(r)$ derived by Fagan et al. (1999) will deliver a distinction between these classifications. So it is very much necessary to put an environmental restriction delineating a correlation between the species $I G R$ and the external fluctuation.

The application of the stochastic calculus upon the model 5.5 provide an environmental bound to the density regulation. Since the contribution of all the parameters is evident in the growth of any species, so the bound should be a function of all the growth parameters. We found from the theorem 5.1 the analytical expression of such kind of environmental restriction. The relation (5.6) depicts that the environmental noise intensity has a strong positive correlation with the species IGR $(r)$. The negative association between the density regulation and the environmental parameter $(\sigma)$ elucidates that the low density regulated species have a chance to dispute with the more level of external fluctuations. Moreover, the carrying capacity is also inversely related to environmental disturbances, i.e., it is quite challenging for the species with a small amount of carrying capacity to sustain in a system with a high level of environmental fluctuation. It is relatively infrequent that the large carrying capacity species can better withstand the external noise (Lande et al., 2009). Although the categorization of the species dynamics by Fagan et al. (2001) is regulated by these characteristics, the relation between the external noise and the species IGR described by the author lacks the necessary information about the carrying capacity and the density regulation.

\section{Data}

In this article, we use the population abundance data of different species from the Global Population Dynamics Database (GPDD)(NERC Centre for Population Biology, 2010). Fegraus et al. (2005) delineate that the GPDD is the most extensive database with nearly five thousand time series data of various 
species throughout the world. This metadata contains the population time series of 22 taxonomic classes viz. (i) Aves; (ii) Mammalia; (iii) Insecta; (iv) Osteichthyes; (v) Reptilia; (vi) Crustacea; (vii) Gastropoda; (viii) Cephalaspidomorphi; (ix) Angiospermopsida (Dicotyledoneae); (x) Angiospermopsida (Monocotyledonae); (xi) Bacillariophyceae; (xii) Coniferopsida; (xiii) Dinophyceae; (xiv) Scyphozoa; (xv) Echinoidea; (xvi) Polychaeta; (xvii) Bivalvia; (xviii) Chondrichtyhes; (xix) Arachnida; (xx) Rotifera; (xxi) Cyanophyceae; (xxii) Amphibia. Note that the most abundant groups present in the GPDD are the first four classes of the above classification (91.4\%). Consequently, like most of the authors (Fagan et al., 2001; Sibly et al., 2005; Bhowmick et al., 2015), we incorporate the time series data of these first four taxonomic classes in our analysis.

\subsection{Data filtration}

We have already mentioned that Sibly et al. (2005) used 1780 population time-series data from the GPDD database to study the density regulatory behavior on the species growth dynamics. The analysis shows that the fitness profile of most of the species (1585 time series, i.e., $89.04 \%$ ) follows the concave upward trend with $\theta<1$. So, we plan to incorporate these time series datasets in our analysis. However, $934 \&$ 124 time-series data remain with the negative and zero estimates of the density regulatory parameter, respectively. The study of Sibly et al. (2005), and Bhowmick et al. (2015) reveal that the negative estimate of any growth parameter leads to the immediate extinction of the population. Moreover, the zero estimates of the curvature parameter turn the growth profile of any species in an unbounded fashion, which does not meet the criteria of density regulation. So, we neglect these datasets for further calculation. Note that in the rest of the 527 time-series, we observe that there exist 7 cases with GPDD ID 1389, 6162, 6320, 6378, 6511, 6649, 6875, whose growth profile is much more synergistic with the cooperative attitude than the theta-logistic trait (Bhowmick et al., 2015). Consequently, in the remaining 520 time-series, we again find 19 datasets representing the taxonomic classes other than the Mammals, Aves, Insects, and Bony fishes. As we mentioned above, these four taxonomic groups are most abundant, so we proceed with our whole analysis with the 501 time series from the database. The summary of this whole filtration process is present in the table 1 of the supplementary file.

\section{Results}

\subsection{Estimation process}

The estimation process associated with the discrete-time analog becomes difficult due to the non-explicit nature of the conditional dynamics in the sampling process of SDEs. So, applying standard techniques like grid-search, least square method, etc., is quite tough for pursuing the regression work with the SDE. In this connection, we need to construct a different framework that will delineate the estimation process when the underlying system becomes stochastic. However, any SDE can be generally expressed by

$$
d X(t)=f(t, N(t), \underline{\theta}) d t+g(t, N(t), \underline{\theta}) d W(t), \quad t \geq 0, N(0)=N_{0}
$$

Here $f: \mathbb{R} \times \Theta \times[0, T] \rightarrow \mathbb{R}, g: \mathbb{R} \times \Theta \times[0, T] \rightarrow \mathbb{R}^{+}$represents the corresponding slowly varying continuous component, i.e., the drift coefficient and the rapidly fluctuating continuous random component, i.e., the diffusion coefficient of the $\operatorname{SDE}$ (7.1) respectively. Note that the unknown component $\Theta(\subseteq \mathbb{R})$ is the parameter space, which needs to be estimated. However, we find a list of authors who put their great effort into developing different estimation processes for the stochastic setup (Dacunha-Castelle and Florens-Zmirou, 1986; Dohnal, 1987; Gallant and Long, 1997; Nicolau, 2002, 2004; Alcock and Burrage, 2004; Uchida and Yoshida, 2005; Brouste and Iacus, 2013). 
Here we use R software (R Core Team, 2019) with the version 3.6.1 for pursuing the whole estimation process. A couple of packages already exists in this software to perform the estimation process. We enlist the name of these packages with their functional properties in the table 1 . In our case we choose the package Sim.DiffProc from this list. Note that it does not approximate the transition density function of (7.1) (Guidoum and Boukhetala, 2020) rather the sample path $N(t)$ is being approximated in such a way that the discrete analog will produce a precise likelihood function.

Table 1: List of packages in $\mathrm{R}$ software to perform the estimation process in respect to the stochastic differential equations.

\begin{tabular}{lll}
\hline Package & Functional property & Reference \\
\hline sde & Package of simulation and infer- & Iacus (2016) \\
ence for SDEs & \\
Buima & $\begin{array}{l}\text { Better for the simulation and } \\
\text { inference for multidimensional }\end{array}$ & Iacus et al. (2017) \\
& SDE & \\
Sim.DiffProc & $\begin{array}{l}\text { Estimation process is con- } \\
\text { ducted by constructing pseudo- }\end{array}$ & (2020) \\
& maximum likelihood method by & \\
& the function fitsde & \\
\hline
\end{tabular}

Here the fitsde function is acting like a precursor in implementing the pseudo-maximum likelihood estimator (henceforth, PMLE) through the Sim.DiffProc package. The principal arguments require to drive the fitsde function is the univariate time series object (ts) and the initial guess (start) for commencing the process. The dataset collected from the GPDD database represents a discrete timeseries object sampled over different time windows. Now, the estimation process is being conducted by the routine pmle, where one needs to specify the underlying method. The standard methods exist to pursue this process are the Euler (Yoshida, 1992; Florens-Zmirou, 1989), Ozaki (Ozaki, 1992), ShojiOzaki (Shoji and Ozaki, 1998), Kessler (Kessler, 1997), etc. We implement four of these methods in the estimation procedure. As a consequence, the output returns the magnitude of the estimated model parameters for all of these mentioned processes. Now we require a standard model selection criteria to select the best estimate. Here we use Akaike Information Criterion (AIC) as a statistical measure for the model selection purpose, which is being reconstructed by Uchida and Yoshida (2005) for the diffusion processes. Note that the outcomes of this research preserve the property of AIC, i.e., the lower the AIC value, the better will be the model. In our case, we initially started this estimation process with 501 time-series data. However, the convergence of the PMLE takes place for only 447 time-series data, i.e., the execution of the estimation process runs smoothly for $89.22 \%$ of the whole datasets. We mention the estimated values of the model parameters for 447 time series in the supplementary material.

Remark 3. We identify quite a few time series for which the model parameters show an unreliable estimate. It means at least one of the model parameters among $r, K, \theta$, and $\sigma$ provide negative estimates, which is absurd from both the mathematical and ecological perspectives. We highlight those datasets with the GPDD ID 6471, 6497, 6503, 6508, 6678, 8471, 8573, 8653, 9215, 9488, 9856 in the supplementary material.

\subsection{Prediction of the extinction probability by the simulation process}

\subsubsection{Mechanisms of species extinction}

Theoretically, null population size is defined as the extinction state of the species. However, the definition of extinction has different variants in the domain of ecology. In the present discussion, we will explore 
this classification with clarity (i) The off-spring production of a species will be impossible to achieve a population size one. The population size with unity ultimately compels the species to an extinction state as mentioned by several authors (Saha et al., 2013; Sau et al., 2020; Sæther et al., 1998). (ii) Allee effect in population can also be treated as one of the extinction vortices of the species. The fitness of the species must be negative when the population abundance falls below a critical threshold (Allee threshold) value (Courchamp et al., 2008; Saha et al., 2013). The obvious consequence of this event is population extinction. This phenomenon is known as strong Allee effect in ecology. (iii) The seminal contribution by Rosenzweig (1971) and many subsequent studies (Huffaker et al., 1963; Luckinbill, 1973; Veilleux, 1979; Fussmann et al., 2000) suggest that an enrichment that is perceived as beneficial for the growth of populations might have the potential to cause destabilization of the ecosystem and eventually extinction of the predator-prey populations. (iv) Last but not least, environmental fluctuation is the most vital component for population extinction, where the population size can be reduced drastically due to sudden unexpected natural calamities (Lande, 1987, 1993). We have described the above four vortices by a flowchart as depicted in figure 4 .

We define the extinction concept from the facet of the simulation process. We generate the population time series for a future time window based on a simulation experiment's history data. We proclaim the species is on the verge of extinction; if at least $50 \%$ cases from the entire time series, the population abundance falls below the initial population size of the cohort.

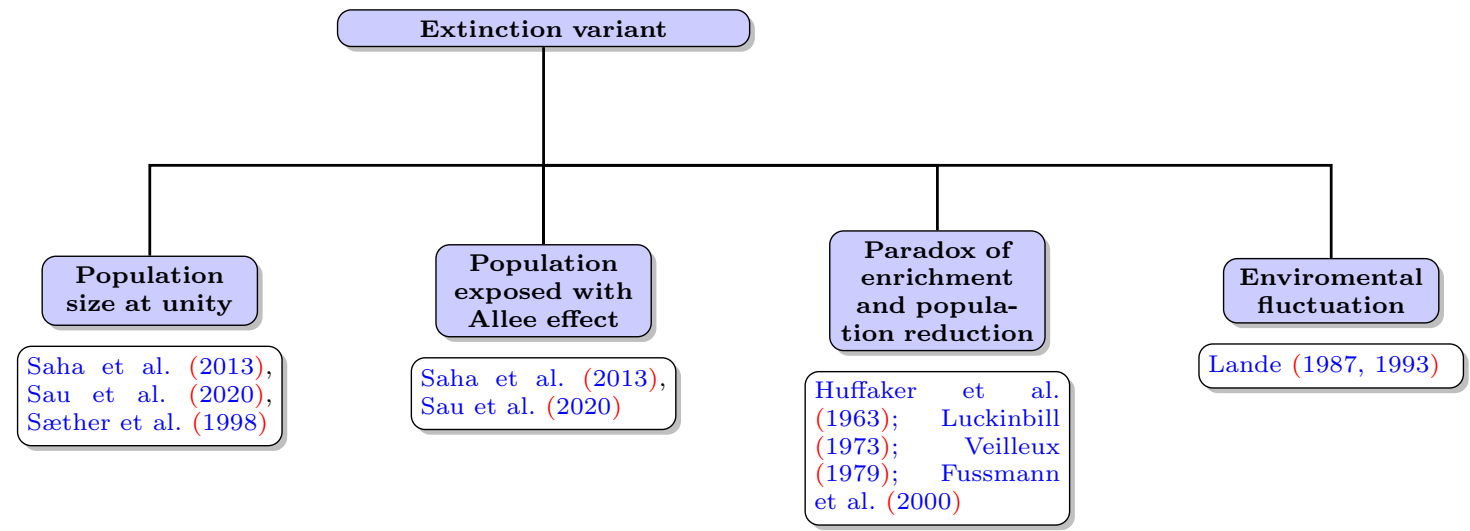

Figure 4: Flow diagram describing different ways of extinction.

\subsubsection{Proposed simulation algorithm and probability of extinction}

We consider very few data among 447 time series to pursue the simulation work. The selection process is manifested by the magnitude of the percentage of variation (henceforth, PV). This measure is defined as the percentage increment of the theoretical magnitude of the noise intensity parameter $\left(\sigma_{t h}\right)$ from its estimated value $(\hat{\sigma})$. Here, we use the theorem 5.1 to enumerate the magnitude of $\sigma_{t h}$. Note that the species will be in relatively safer zone, if $\hat{\sigma}$ does not exceed the theoretical bound, $\sigma_{t h}$ (i.e. $\hat{\sigma} \leq \sigma_{t h}$ ). Therefore, those species now became the cause of concern for which this relationship is disrupted. Hence, we continue our further analysis with all these species datasets.

Here, we observe that for each of the four taxonomic classes, the PV range is too wide between the theoretical and estimated magnitude of $\sigma$. So, we partitioned the range of PV into three categories, i.e., 


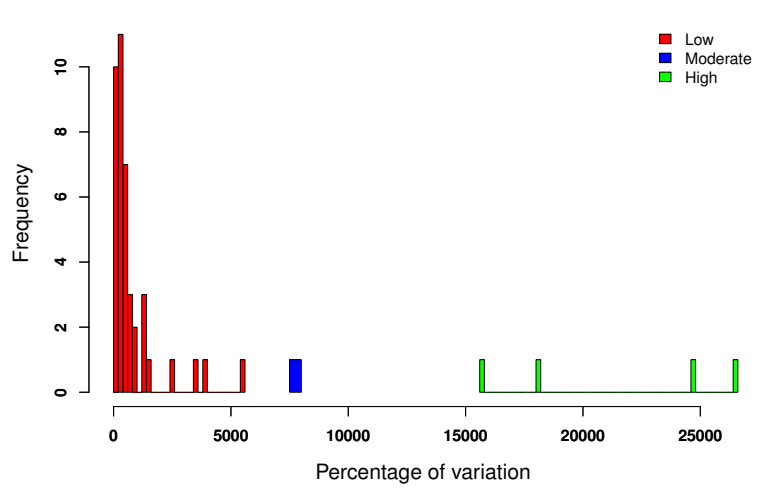

(a)

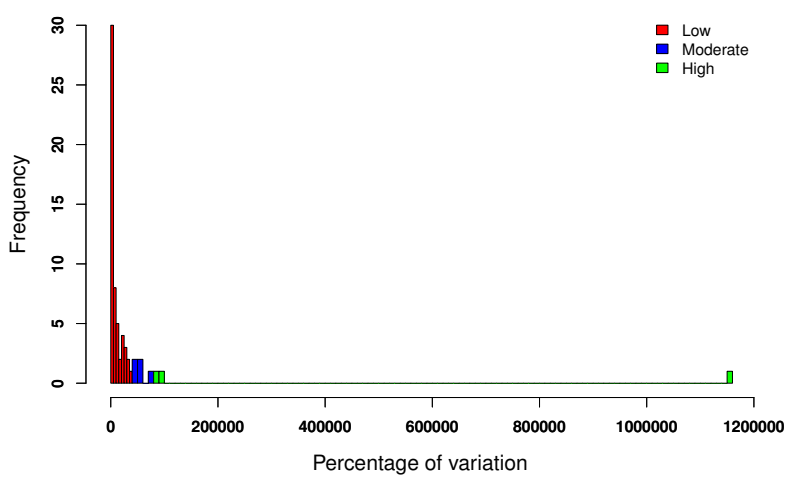

(c)

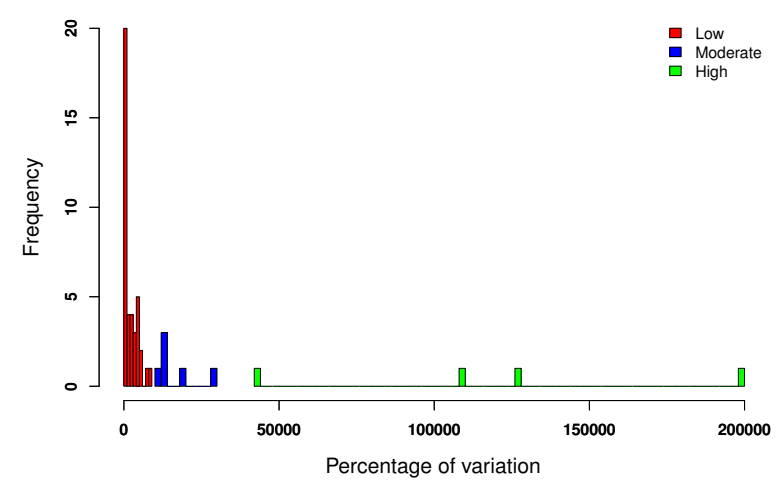

(b)

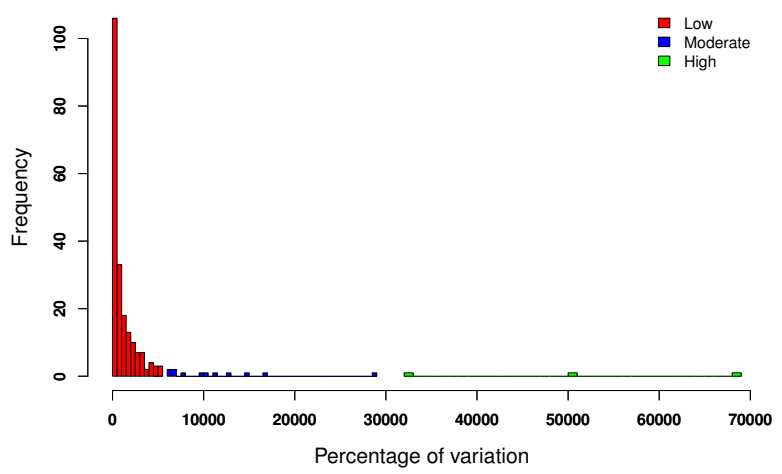

(d)

Figure 5: Probability distribution of the percentage of variation for the taxonomic classes (a) Aves; (b) Mammal; (c) Fish; (d) Insect, respectively.

(i) low range of variation; (ii) moderate range of variation; (iii) the high range of variation. We mention this classification in the figure 5. However, it is quite clear from the classification that the species belong to the last two clusters will be more prone to extinction than the first category. The deviation between $\hat{\sigma}$ and $\sigma_{t h}$ is playing the major role. In this connection, we consider an interval $I=\left[a_{P V}, b_{P V}\right]$ to select those species dataset, where $a_{P V}$ denotes 0.5 percent of the maximum PV, and $b_{P V}$ indicates the maximum percentage of increment. Thus, the time series should be selected so that the PV magnitudes should belong to the last two classifications and that particular interval $I$. Hence, the number of datasets is now being reduced to 71 , i.e., $15.92 \%$, which are on the verge of extinction.

The GPDD species abundance data were collected on different time windows. Overlapping windows with a wider span are hardly available, so comparing extinction status based on non-overlapping time windows is not meaningful. To check the overlapping and non-overlapping time windows, we have drawn a diagram depicted in figure 6 . From this figure, we can conclude that the assessment of extinction status based on the Insect data is meaningful compared to other taxonomic groups. A significant overlapping period between 1970 to 1990 is available in the GPDD database for the Insect community. To overcome this problem for the different taxonomic groups, we will propose a simulated prediction model for deter- 
mining the extinction status of the species for the current time period (2021). Note that the simulation process will now be manifested on the above 71 time-series data. The protocol of the simulation experiment is given into the following:

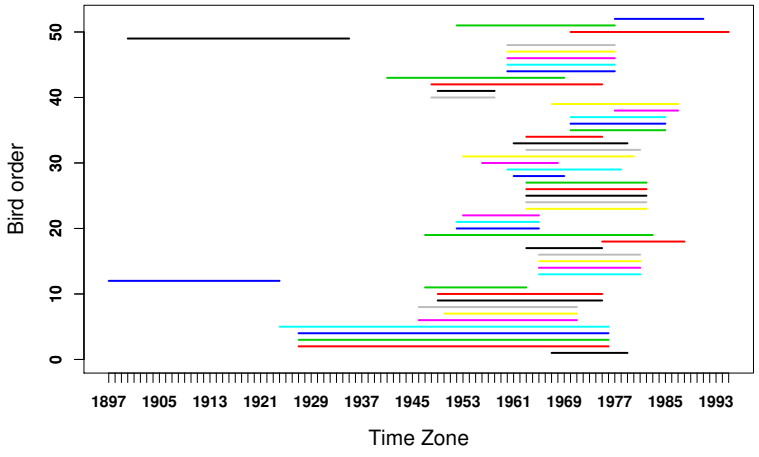

(a)

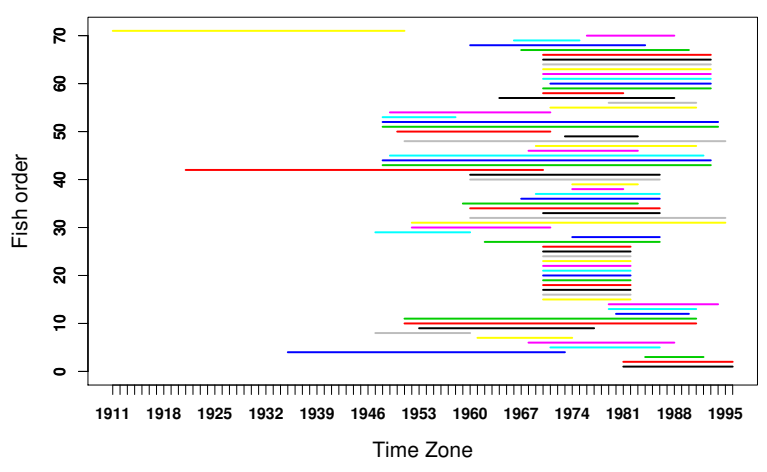

(c)

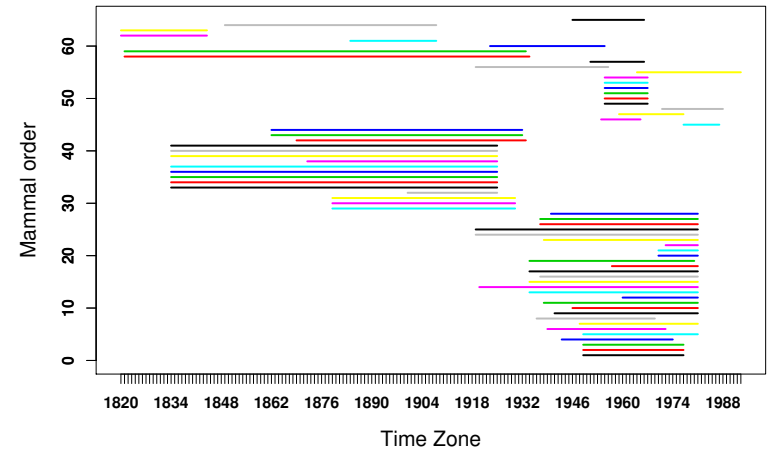

(b)

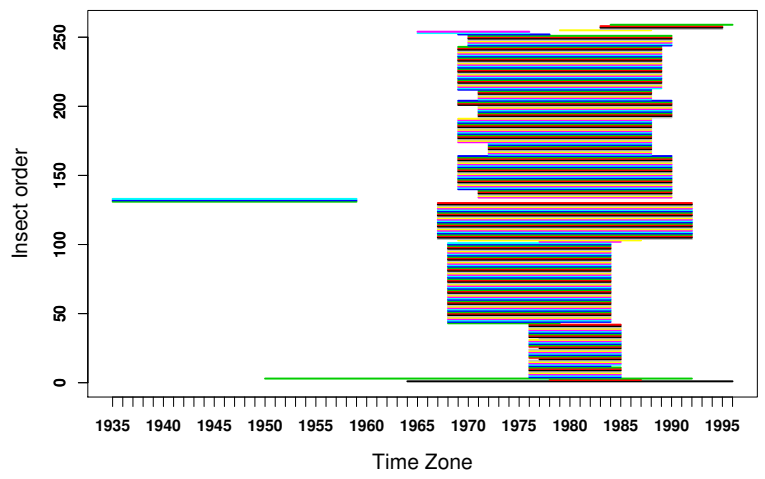

(d)

Figure 6: Time windows.

1. Generate a time series data for $q$ time points following the growth equation 5.2, where $q=\left|2021-t_{f}\right|$ and $t_{f}$ be the year up to which the data is collected.

2. Repeat the process for $m$ times to generate a $m \times q(m>q)$ order matrix, where each row represents a single sample-path.

3. Check the "extinction status", as described in the section 7.2.1 for each of the $m$ realizations.

4. Identify the realizations for which our rule of "extinction" is applicable and mark them by number 1 and others by 0 .

5. Let $p$ denote the number of cases marked by 1 . So, the probability of extinction (henceforth, PE) for these $m$ realizations is $\frac{p}{m}$.

6. Repeat the process 1-5 for $s(>m)$ times to generate a distribution of PE. 
7. Finally, we have identified the mean of this distribution as an estimate of PE for any particular time series data.

\section{Discussion}

The dynamical relationship between the population density and its fitness can be well understood by the density regulation in any species growth (Sibly et al., 2005, 2007; Ross, 2009; Bhowmick et al., 2015). The classical growth model describing the density dependence is the logistic growth equation. However, the linear decrement of relative growth rate (henceforth, RGR) with the increasing population size put a restriction on the explanation of density dependence through the logistic growth law (Ross, 2009). In this connection, Sibly et al. (2005) put a significant effort into justifying that the species inherent growth dynamics can not be well described by this linear association. The authors explain that the fitness profile of most of the species is depicted through the non-linear structure (i.e., either convex or concave) depending upon its density regulation. The empirical study of Sibly et al. (2005) delineates that the density regulation in most of the species is maintained through the theta-logistic model. The authors described that most species have a fundamental characteristic to show the non-linear fitness profile with a convex downward trend. Consequently, it seems that at the low population density, the fitness level of the species is going to zero. The species then have to produce more offspring to reach their asymptotic size.

Note that the research work of Sibly et al. (2005) is demonstrated based on the continuous setup. However, we have already mentioned in section 2 that in any ecosystem, two types of growth generations are observed, i.e., overlapping and non-overlapping. The first one can be expressed through the continuous growth equation and the latter with the discrete growth law. Since we focus on the theta-logistic growth regulation besides the continuous system, we must spotlight the discrete growth dynamics. So, we construct the section 4 , where we take the consortium of bifurcation analysis to describe the inherent growth mechanism of the discrete growth law. The characteristics of any discrete system can be well understood through the bifurcation analysis. In this connection, we propose the theorem 4.1 to elucidate the intricate property of the discrete theta-logistic growth dynamics. The numerical simulation on this discrete map produces the bifurcation diagram 1, which shows the attainment of all the phases, i.e., 2-period cycle, limit cycle, chaos, etc. of the discrete theta-logistic dynamics. In this diagram, we consider Oncorhyncus gorbuscha as a testbed species to describe the bifurcation analysis. Note that these cases' consideration is driven by choice of IGR and density regulated parameter values. Since the study of Clark et al. (2010) reveal that the two parameters $r, \theta$ can not act as the independent parameters in any growth law. Instead, the compilation of these two parameters will always provide the necessary information about the species asymptotic size as well as the stability of the concerned system (Saha et al., 2013; Kundu et al., 2018). Note that the increase in the density regulation leads any species towards the Malthusian growth. Consequently, if the species do not optimize their offspring production rate, a chaotic situation will generate due to the limited resources. Hence the species have to minimize the IGR so that stability is maintained throughout the ecosystem. The figure 1 elucidates this scenario. It is shown that if the IGR values are increased with the enhancement of the density regulation, the chaotic regime will be found in the discrete growth dynamics, leading any species to extinction.

Moreover, a hump has been formed for each period-doubling limit cycle case in the case of the bifurcation diagram of the discrete one-dimensional map. This period-doubling limit cycle ultimately converts to a chaotic regime in the long run while the bifurcation diagram progresses. Such period-doubling limit cycles are visible in figure 1 . The natural question arises whether the bifurcation diagram remains 
unaltered if we introduce the noise in the deterministic system. For illustration, we have chosen a fish Oncorhyncus gorbuscha, which is known to be a species with non-overlapping generation due to its anadromous character (Kot, 2001). Hence the discrete theta-logistic model is appropriate to describe the growth profile of the species. So the bifurcation diagram concerning the growth parameter IGR is depicted in figure 2 under stochastic setup. The diagram exhibits that under the stochastic environment, the stable character of the species growth dynamics is maintained up to a comparatively high magnitude of IGR $(r=3.1)$ than the deterministic process. Moreover, just one hump is visible in the stochastic case compared to multiple humps of deterministic setup, which implies that species are more prone to reach the chaotic zone under the noisy interaction quickly. Moreover, when we increase the noise intensity by $50 \%, 75 \%$, and $90 \%$, the species rapidly moves towards the chaotic state (see panel (b), (c), (d) of figure 2). So there is a strong association between the chaotic regime and the environmental variation.

\subsection{Probability of extinction and population viability analysis}

\subsubsection{Different avenues of population viability analysis}

Population Viability Analysis (henceforth, PVA) is one of the popular methods in ecology to explore the species extinction status under some specified conditions. Several ecologists acknowledge the escalating role of PVA in species conservation. The concept of PVA initially comes from the perception of minimum viable population size (henceforth, MVP). It is assumed that the manifestation between MVP and PVA is first noticed in the work of Shaffer (1981). The author used the stochastic population dynamics simulation model with certain demographic structures to enumerate the risk of extinction and the MVP for the Grizzly Bears. However, the pros and cons of the PVA are first noticed in the article of Boyce (1992), where the author discuss necessary components to drive the PVA. Later many authors put a significant effort in providing a proper shape to the concept of PVA.

The scrutiny of this literature depicts that the role of PVA in predicting the species extinction status is still a debatable issue due to its level of accuracy. The study of Taylor (1995), Brook et al. (1997), Reed et al. (1998) and Ludwig (1999) show that the data deficiency and the presence of ambiguity over the population structure may sometime lead to the unacceptable results in the population persistence. On the contrary, Brook et al. (2000) performed a retrospective analysis using 21 wildlife populations and concluded that the outcomes of PVA are surprisingly accurate. Note that this study is being provoked to criticism due to the usage of short time windows. Apart from this, Schiegg et al. (2005) also showed the predictive accuracy of PVA by the population abundance data set of red-cockaded woodpecker $(P i$ coides borealis). The authors found that the predictability of PVA turned out to be accurate in a short period of five years. Consequently, Crone et al. (2013) discovers the poor accuracy level of PVAs due to the presence of variation in the environmental conditions in between the data collection and forecasting periods. So, it is recommended to include the environmental changes in the forecasting model structure for better accuracy. In this connection, several authors, viz., McCarthy et al. (2001), Münzbergová and Ehrlén (2005), Pe'er et al. (2013), etc. suggest a list of methods to enhance the accuracy level of PVA. Note that, whatever be the degree of precision, the analysis of PVA is conducted based on the computer simulation (IUCN, 2019).

IUCN has summarised five criteria (A to E) for evaluating the threatened status of many species. These criteria are based on the population reduction, the extent of occurrence, areas of occupancy, the decline of small populations, number of mature individuals in declining tiny populations. Finally, a quantitative computer-based simulation is an essential tool for predicting the species threatened status. 
The application of PVA is noticed in species conservation by evaluating the extinction probability of any species within a specified time window. This enumeration method in PVA depends on the two major components, i.e., (i) Data availability, (ii) Ecology of the species. Münzbergová and Ehrlén (2005) shows that the output of PVA can create a wrong impression on the species conservation due to the inappropriate collection of the datasets. In this regard, the authors propose a method describing that the sampling of the data set at any stage of the species life cycle should be identical for the better accuracy of PVA outputs. In addition, the ecology of the species also plays a key role in providing a precise estimate of PVA, which the model selection criteria can well demonstrate. IUCN prescribed five different types of mathematical models to nurture the species extinction norms, viz., (i) occupancy model (Sjögren-Gulve and Hanski, 2000), (ii) scalar dynamic (unstructured) model (Dennis et al., 1991; Burgman et al., 1993), (iii) stage-structured model (Akçakaya, 2000), (iv) individual-based model (Lacy, 2000), and (v) metapopulation model. Our entire work is designed on the scalar dynamic model structure.

Dennis et al. (1991) proposed a method of estimation of growth and extinction parameters for endangered species based on the stochastic exponential growth model. This seminal contribution is the first systematic and analytical study of population viability analysis using sophisticated statistical concepts and tools. However, most of the species growth rate is density-dependent, so the choice of exponential model is hardly a likely event in reality. The same author has used an alternative statistical computing method, called data cloning, to calculate the maximum likelihood estimates and its standard errors for complex ecological models (Lele et al., 2007). Although the vital component of the method involves the Bayesian setup for MCMC computation, this framework is only used as a tool for the likelihood calculation. In contrast to the classical Bayesian analysis, the inferences are entirely invariant to the choice of the prior distributions.

Several software packages exist, viz., GAPPS, INMAT, RAMAS/AGE, VORTEX, etc., to explore the population viability analysis in recent times. However, there has not been any systematic attempt to compare predictions by several softwares based on a single data set. There has been a significant change in the output of PVA analysis if we use the density dependence model for species growth with demographic and environmental stochasticity. Nevertheless, it is essential to note that the modeler has no idea what is going on in software packages for proposing the result of any study. This is typically known as the black box syndrome.

\subsubsection{Extinction probabilities of the selected species}

It is already mentioned in section 7.2.2 that based on the standard data filtration and simulation process, we choose 71 time-series profiles for describing their conservation status. There are 5 Aves, 19 Insect, 15 Mammals, and 31 Fish data present among these profiles. We include our prediction results about this set of species in the table 2-7 of the supplementary file. These tables are formulated to serve two significant purposes. The first one is the enumeration of the probability of extinction of these species concerning 2021, which are enlisted in the fifth column of each table. The second one is the categorization of the species according to the IUCN guidelines. It is worthwhile to mention that there present five criteria in IUCN, i.e., criteria A, B, C, D, and E, respectively, to predict any species conservation status. All these criteria except the last one are generally enumerated based on the species demographic characteristics, area of occupancy, habitat place, etc., which are available in many kinds of literature. However, criterion E is overlooked in most of the studies. The plausible reason for this ignorance is the quantitative analysis, which is the fundamental characteristic of criterion E. This conservation measure is also subdivided into three labels, i.e., Endangered (EN), Critically Endangered (CR), and Vulnerable (VU). The guidelines of these three labels are present in the IUCN report. We also marked some 
species with these labels and put them into the 8th column of table 2-7 present in the supplementary file.

Note that IUCN does not include any invertebrates like insects in the conservatory analysis. Recently Fox et al. (2019) prepared a list of insects by labeling the conservatory position according to IUCN protocol. However, our enlisted insects in table 3 (see the supplementary file) do not overlap with the set of species mentioned in the article of Fox et al. (2019). So, we prepare such kind of list in the table 3 of the supplementary file for the Insect data following the IUCN guidelines. In our entire analysis, we observe $40 \%$ cases where our prediction regarding the conservatory label meets the IUCN result, and in $60 \%$ cases, it does not. We also make a column at the end of the table in to elucidate this scenario except for the aforementioned table 3. Here we use two abbreviations, i.e., "M" for the species where our result matches IUCN and "MM" for the mismatch cases.

Remark 4. The species abundance data in GPDD are pretty old to 2021. So, the prediction about the current conservatory status of any species would be more accurate if the time gap between the final calendar year of any time-series profile and 2021 is reduced. In this connection, we perform the cluster analysis of the 71-time series to explore several compartments of the difference in the time windows $(D T W)$. The output of this analysis is present in supplementary file, where the GPDD IDs are present at the single branch of this dataset. The figure shows the different groups of DTW among which we select the cluster with a reasonable magnitude of DTW, i.e., 50. We also make a column in each table 2-7 of the supplementary file by providing the header "Reliability percentage" under which we keep the $D T W$ value along with the deviation percentage from level 50. Note that we do not provide any reliability percentage in the table 5 of the supplementary file due to the sizeable DTW magnitudes as the prediction process becomes cruder with the increasing DTW level.

Remark 5. We got the estimate of the noise intensity parameter $(\hat{\sigma})$ for the aforementioned 447 timeseries data. We draw the frequency histogram 8 of these estimated magnitudes according to the taxonomic class, i.e., Aves, Mammal, Fish, Insect, respectively. The diagram reflects that the tolerability level of the noise intensity is not too high for these four groups of species. However, there are some variations in these tolerance levels. The figure shows that the taxonomic class Aves possess the lowest tolerance level, and the Insect is the highest among these four. So, suppose we prepare a rank list about these tolerance levels. In that case, it should follow the trend of Insect, Fish, Mammal, Bird respectively.

We enumerate the extinction probability of the selected 71 time-series profile by our proposed simulation method as mentioned in the section 7.2.2. These extinction probabilities are attached at the fifth column of the tables 2-7 of the supplementary file. The figure 7 depicts the histogram of these probability magnitudes for three major taxonomic groups, i.e., Insect, Fish, and Mammals. Note that in the case of the selected birds, the probability values turn out to be zero; that is why we exclude the Aves group from the histogram 7 (see table 2 in the supplementary file). The diagram exhibits that the extinction probabilities can be distinguished into two distinct zones like low and high, in the case of Fish and Mammal. However, for the Insect, we observe low, moderate, and high-risk zones about the probability magnitudes. It is worth mentioning that the high extinction probability certainly significantly raises any species' risk status. In this connection, we choose at most three species from the Insect, Fish, and Mammals with the high extinction probability to discuss their conservation management. But, in the case of the taxonomic class Mammal, we find only one such species. 


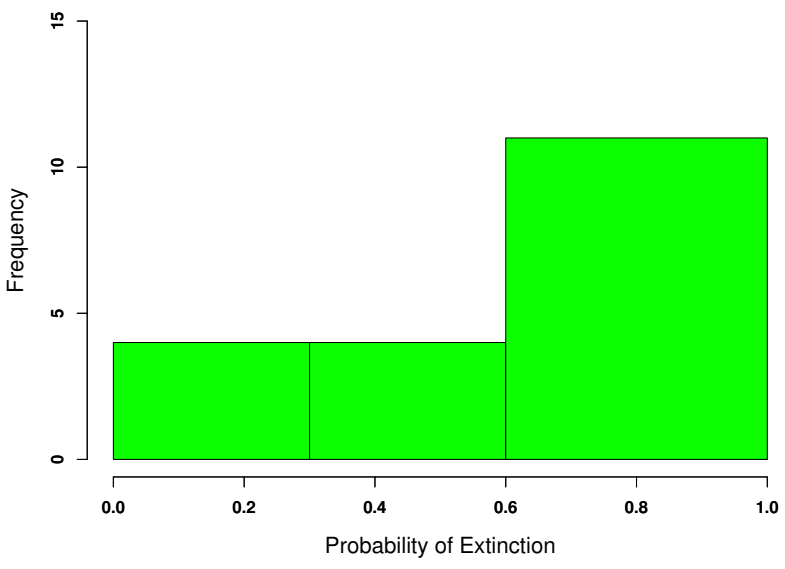

(a)

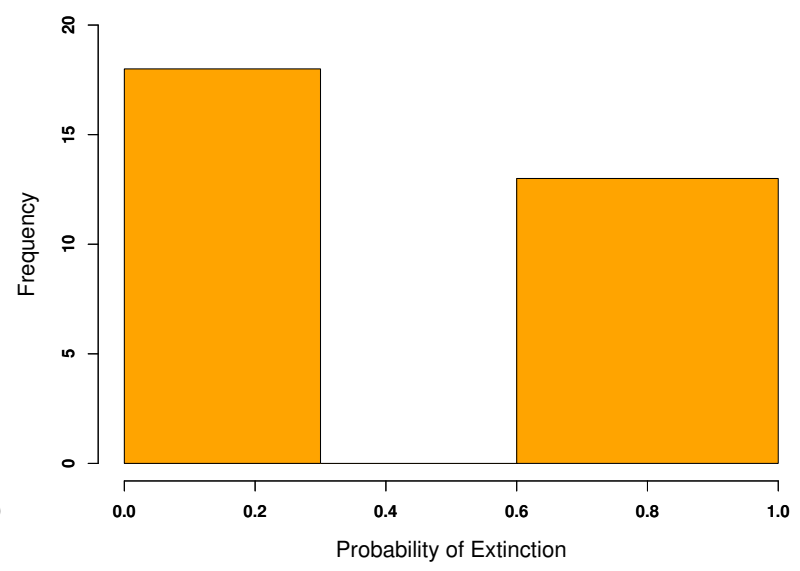

(b)

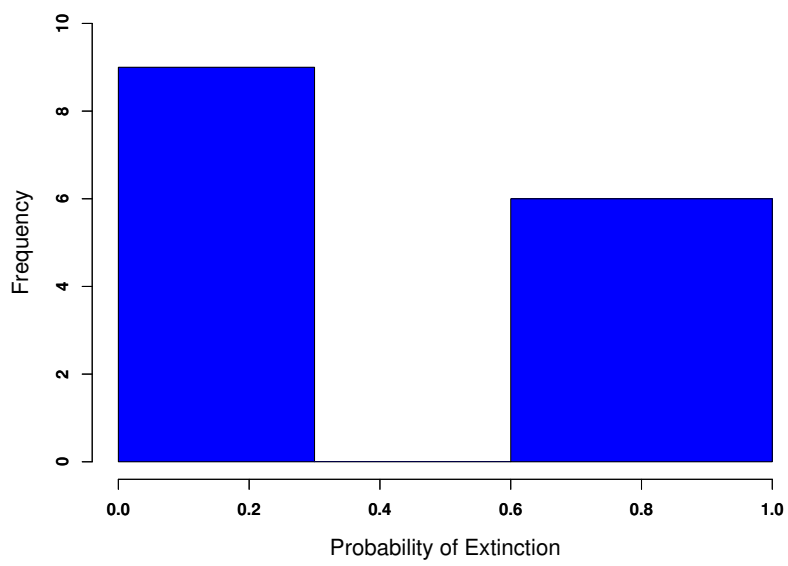

(c)

Figure 7: The frequency distribution of the estimated probability of extinction to 2021, for the three major taxonomic groups, i.e., (a) Insect, (b) Fish, and (c) Mammal. 


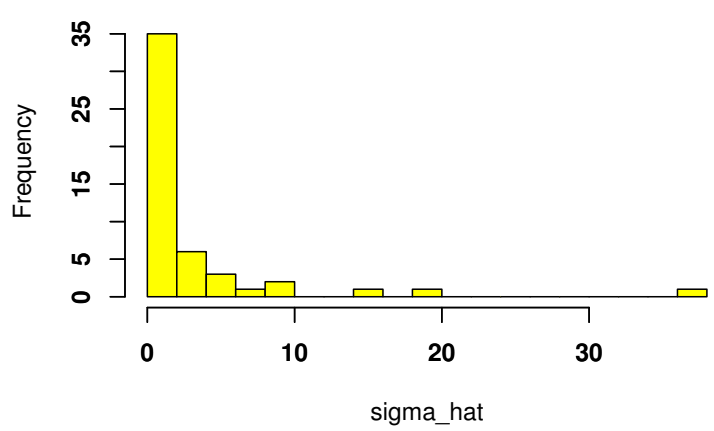

(a) Aves

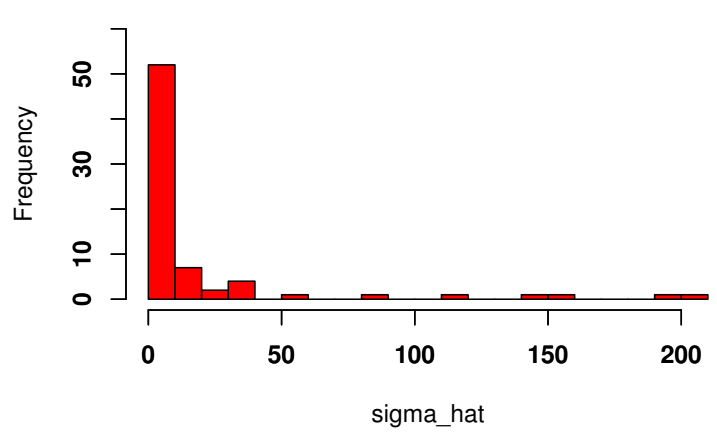

(c) Fish

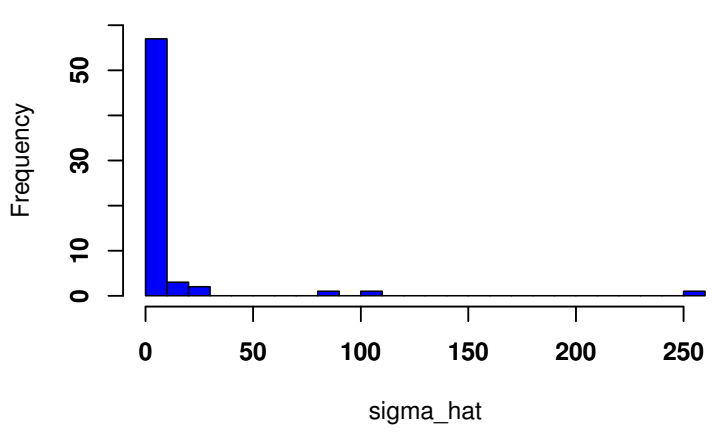

(b) Mammal

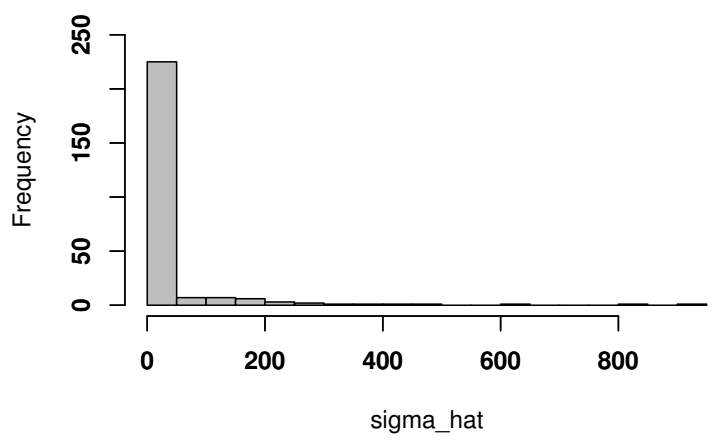

(d) Insect

Figure 8: Frequency distribution of the estimated magnitude of noise intensity parameter $(\hat{\sigma})$ for (a) Aves, $(\mathrm{b})$ Mammal, (c) Bird, (d) Insect.

The taxonomic name of each selected species is present in the second column of the table 2 . In order to assess the effectiveness of our prediction model in the conservation scheme of any species, we provide a detailed discussion on the population abundance of these selected species in several provinces of the world. This is given in the supplementary file. Based on this analysis, we also prepare a column in the table 2, mentioning the conservation guidelines of each species. It is needless to say that the dataset of Metapolophium festucae in the GPDD is collected from the Hereford and Worcester provinces of the United Kingdom. However, we do not find any report about the abundance of this insect in the United Kingdom to support our findings. We also observe different PE values of any individual for the dataset of distinct zones. As a consequence, a variation is generated in the conservation status of any particular species. The taxonomic names of such species are Oncorhyncus nerka, (Sockeye salmon) and Solea vulgaris (Dover sole) with the GPDD ID 2019, 2041 respectively. So, we exclude these two IDs from the third column of the table 2. Although Sockeye salmon stands out to be the highly-threatened species among several places of British Colombia and Alaska, the extinction probability has been zero for the dataset of Stellako River. The survey of Welch et al. (2007) on the Stellako River support this fact. Similarly, in the case of Dover sole, we observe a high extinction-level at the ICES VIID, North Sea region. Nevertheless, the species is abundant in the Bay of Biscay. Koutsikopoulos and Lacroix 
Table 2: Conservation guidelines for the species with high extinction threat.

\begin{tabular}{|c|c|c|c|}
\hline Taxonomic class & Species name & GPDD ID & Conservation scheme \\
\hline Insect & $\begin{array}{l}\text { Phorodon humuli } \\
\text { Metapolophium fes- } \\
\text { tucae } \\
\text { Drepanosiphum } \\
\text { platanoidis }\end{array}$ & $\begin{array}{l}8437 \\
8430 \\
8153\end{array}$ & $\begin{array}{l}\text { The pesticide should be used in a controlled } \\
\text { fashion. } \\
* \\
* *\end{array}$ \\
\hline Fish & $\begin{array}{l}\text { Oncorhyncus nerka } \\
\text { Solea vulgaris } \\
\text { Sprattus sprattus } \\
\text { Oncorhyncus gor- } \\
\text { buscha }\end{array}$ & $\begin{array}{l}1974,1978,1999 \\
2039 \\
6550\end{array}$ & $\begin{array}{l}\text { Use of pathogens should be reduced so that } \\
\text { species can grow in a natural way. } \\
\text { Overexploitation or Overfishing should be } \\
\text { controlled. } \\
\text { The oceanographic parameters especially the } \\
\text { water temperature of the habitat place should } \\
\text { be monitored. } \\
\text { Monitor this fish regularly at a few regions } \\
\text { of Alaska, i.e., the Auke creek, Post Gra- } \\
\text { ham, Rockey River and that of Upper Skeena, } \\
\text { Central Coast Area of the British Colombia, } \\
\text { Canada. }\end{array}$ \\
\hline Mammal & Castor canadensis & 198 & $\begin{array}{l}\text { Hunting of species should be restricted and the } \\
\text { reintroduction of species is needed. }\end{array}$ \\
\hline
\end{tabular}

* We do not find the species abundance report of Metapolophium festucae in United Kingdom.

** The species is very sensitive to the environmental fluctuations so the species need special care but this task is not easy at all. A further thorough and deep study would be beneficial to identify the management actions for the conservation of the species. The conservation biologists can explore this in future.

+ The fish Pink Salmon has the anadromous character, which is synergistic with the non-overlapping generation;

(1992) have a closed parity with our findings for the region Bay of Biscay. So, the predicted probability of extinction for the species Solea vulgaris is naturally low (0.003) in this region. The plausible reason for this kind of disparity is the estimation of the local extinction of many species.

\section{Conclusion}

The study of Sibly et al. (2005) reveals that most of the species follow the theta-logistic growth trait with the convex downward trend. This entire research work is formulated based on the deterministic setup. In contrast, the involvement of external noise in any growth system is inevitable. Here, we frame the theta-logistic model with the stochastic analog in two directions, i.e., the discrete and continuous setup. The discrete model analysis provides that the extinction state's attainment happens more quickly in the case of a stochastic setup than the deterministic version. Although the role of chaos in species extinction is debatable, literature surveys suggest that chaos with stochasticity accelerates the extinction of species. Similarly, in the case of the continuous version, we performed a theoretical study on the stochastic thetalogistic model to provide a critical value of the noise intensity parameter. This environmental bound will act as the sustainability criteria of any species environmental tolerability. In this connection, we use the data of four major taxonomic groups, i.e., Bird, Insect, Mammal, and Fish, from the GPDD database 
and classify the species based on the environmental sensitivity, i.e., low, moderate, and high. The high sensitive species have a low tolerance level, associated with the small magnitude of environmental noise intensity parameter $\sigma$. Moreover, the simulation prediction model on these four taxonomic classes also shows that the overall extinction probability of the Bird is almost negligible for the current time window.

\section{Declaration}

\section{Acknowledgement}

The author Ayan Paul is thankful to the Department of Science and Technology, Government of India, i.e., DST-INSPIRE (Grant Number: IF180793) for supporting the fellowship.

\section{Conflict of interest}

The authors have no conflict of interest.

\section{References}

Akçakaya, H.R., 2000. Population viability analyses with demographically and spatially structured models. Ecological Bulletins, 23-38.

Alcock, J., Burrage, K., 2004. A genetic estimation algorithm for parameters of stochastic ordinary differential equations. Computational Statistics \& Data Analysis 47, 255-275.

Allen, J., Schaffer, W.M., Rosko, D., 1993. Chaos reduces species extinction by amplifying local population noise. Nature 364, 229-232.

Anderson, D., 2013. Introduction to stochastic processes with applications in the biosciences. .

Andrewartha, H., Birch, L., 1984. The ecological web: More on the distribution and abundance of animals.,(the univeristy of chicago press: Chicago, il.). The ecological web: More on the distribution and abundance of animals. The University of Chicago Press, Chicago, IL. .

Arditi, R., Ginzburg, L.R., 1989. Coupling in predator-prey dynamics: ratio-dependence. Journal of theoretical biology 139, 311-326.

Armstrong, J.D., Bean, C.W., Wells, A., 2018. The scottish invasion of pink salmon in 2017. Journal of fish biology 93, 8-11.

Berryman, A., Millstein, J., 1989. Are ecological systems chaotic - and if not, why not? Trends in Ecology \& Evolution 4, 26-28.

Bhowmick, A.R., Bandyopadhyay, S., Rana, S., Bhattacharya, S., 2016. A simple approximation of moments of the quasi-equilibrium distribution of an extended stochastic theta-logistic model with non-integer powers. Mathematical biosciences 271, 96-112.

Bhowmick, A.R., Saha, B., Chattopadhyay, J., Ray, S., Bhattacharya, S., 2015. Cooperation in species: Interplay of population regulation and extinction through global population dynamics database. Ecological Modelling 312, 150-165.

Bishwal, J., 2008. Parameter estimation in stochastic differential equations. Evol. Appl. . 
Boyce, M.S., 1984. Restitution of r-and k-selection as a model of density-dependent natural selection. Annual review of ecology and systematics 15, 427-447.

Boyce, M.S., 1992. Population viability analysis. Annual review of Ecology and Systematics 23, 481-497.

Brook, B.W., Lim, L., Harden, R., Frankham, R., 1997. Does population viability analysis software predict the behaviour of real populations? a retrospective study on the lord howe island woodhen tricholimnas sylvestris (sclater). Biological Conservation 82, 119-128.

Brook, B.W., O'Grady, J.J., Chapman, A.P., Burgman, M.A., Akcakaya, H.R., Frankham, R., 2000. Predictive accuracy of population viability analysis in conservation biology. Nature 404, 385-387.

Brouste, A., Iacus, S.M., 2013. Parameter estimation for the discretely observed fractional ornsteinuhlenbeck process and the yuima r package. Computational Statistics 28, 1529-1547.

Burgman, M.A., Ferson, S., Akçakaya, H.R., 1993. Risk assessment in conservation biology. volume 12. Springer Science \& Business Media.

Chakraborty, B., Bhowmick, A.R., Chattopadhyay, J., Bhattacharya, S., 2017. Physiological responses of fish under environmental stress and extension of growth (curve) models. Ecological modelling 363, $172-186$.

Charlesworth, B., et al., 1994. Evolution in age-structured populations. volume 2. Cambridge University Press Cambridge.

Clark, F., Brook, B.W., Delean, S., Reşit Akçakaya, H., Bradshaw, C.J., 2010. The theta-logistic is unreliable for modelling most census data. Methods in Ecology and Evolution 1, 253-262.

Courchamp, F., Berec, L., Gascoigne, J., 2008. Allee effects in ecology and conservation. Oxford University Press.

Crone, E.E., Ellis, M.M., Morris, W.F., Stanley, A., Bell, T., Bierzychudek, P., Ehrlén, J., Kaye, T.N., Knight, T.M., Lesica, P., et al., 2013. Ability of matrix models to explain the past and predict the future of plant populations. Conservation Biology 27, 968-978.

Dacunha-Castelle, D., Florens-Zmirou, D., 1986. Estimation of the coefficients of a diffusion from discrete observations. Stochastics: An International Journal of Probability and Stochastic Processes 19, 263-284.

Dennis, B., Desharnais, R.A., Cushing, J.M., Henson, S.M., Costantino, R.F., 2003. Can noise induce chaos? Oikos 102, 329-339.

Dennis, B., Munholland, P.L., Scott, J.M., 1991. Estimation of growth and extinction parameters for endangered species. Ecological monographs 61, 115-143.

Desharnais, R., Costantino, R.F., 1983. Natural selection and density-dependent population growth. Genetics 105, 1029-1040.

Dohnal, G., 1987. On estimating the diffusion coefficient. Journal of Applied Probability, 105-114.

Fagan, W., Meir, E., Prendergast, J., Folarin, A., Kareiva, P., 2001. Characterizing vulnerability to extinction for 758 species. Ecology Letters 4, 132-138. 
Fagan, W.F., Meir, E., Moore, J.L., 1999. Variation thresholds for extinction and their implications for conservation strategies. The American Naturalist 154, 510-520.

Fegraus, E.H., Andelman, S., Jones, M.B., Schildhauer, M., 2005. Maximizing the value of ecological data with structured metadata: an introduction to ecological metadata language (eml) and principles for metadata creation. The Bulletin of the Ecological Society of America 86, 158-168.

Florens-Zmirou, D., 1989. Approximate discrete-time schemes for statistics of diffusion processes. Statistics: A Journal of Theoretical and Applied Statistics 20, 547-557.

Foley, P., 1994. Predicting extinction times from environmental stochasticity and carrying capacity. Conservation Biology 8, 124-137.

Fox, R., Harrower, C.A., Bell, J.R., Shortall, C.R., Middlebrook, I., Wilson, R.J., 2019. Insect population trends and the iucn red list process. Journal of Insect Conservation 23, 269-278.

Fussmann, G.F., Ellner, S.P., Shertzer, K.W., Hairston Jr, N.G., 2000. Crossing the hopf bifurcation in a live predator-prey system. Science 290, 1358-1360.

Gallant, R., Long, J.R., 1997. Estimating stochastic daerential equations efficiently by minimum chisquared. Biometrika 84, 125-141.

Golec, J., Sathananthan, S., 2003. Stability analysis of a stochastic logistic model. Mathematical and Computer Modelling 38, 585-593.

Guidoum, A.C., Boukhetala, K., 2020. Sim.DiffProc: Simulation of Diffusion Processes. URL: https: //cran.r-project.org/package=Sim.DiffProc. $r$ package version 4.5.

Heering Jr, T.E., Reed, D.H., 2005. Modeling extinction: Density-dependent changes in the variance of population growth rates. General Article 50, 183.

Henle, K., Sarre, S., Wiegand, K., 2004. The role of density regulation in extinction processes and population viability analysis. Biodiversity \& Conservation 13, 9-52.

Huffaker, C., Shea, K., Herman, S., et al., 1963. Experimental studies on predation: complex dispersion and levels of food in an acarine predator-prey interaction. Hilgardia 34, 305-330.

Huisman, J., Weissing, F.J., 1999. Biodiversity of plankton by species oscillations and chaos. Nature $402,407$.

Iacus, S.M., 2016. sde: Simulation and Inference for Stochastic Differential Equations. URL: https: //CRAN.R-project. org/package=sde. r package version 2.0.15.

Iacus, S.M., Mercuri, L., Rroji, E., 2017. $\operatorname{Cogarch}(p, q)$ : Simulation and inference with the yuima package. Journal of Statistical Software 80, 1-49. doi:10.18637/jss.v080.i04.

Johnson, M.T., Agrawal, A.A., 2003. The ecological play of predator-prey dynamics in an evolutionary theatre. Trends in Ecology \& Evolution 18, 549-551.

Kessler, M., 1997. Estimation of an ergodic diffusion from discrete observations. Scandinavian Journal of Statistics 24, 211-229.

Khasminskii, R., 2011. Stochastic stability of differential equations. volume 66. Springer Science \& Business Media. 
Kot, M., 2001. Elements of mathematical ecology. Cambridge University Press.

Koutsikopoulos, C., Lacroix, N., 1992. Distribution and abundance of sole (solea solea (1.)) eggs and larvae in the bay of biscay between 1986 and 1989. Netherlands Journal of Sea Research 29, 81-91.

Kundu, S., Mukherjee, J., Yeasmin, F., Basu, S., Chattopadhyay, J., Ray, S., Bhattacharya, S., 2018. Growth profile of chaetoceros sp. and its steady state behaviour with change in initial inoculum size: a modelling approach. CURRENT SCIENCE 115, 2275-2286.

Lacy, R.C., 2000. Considering threats to the viability of small populations using individual-based models. Ecological Bulletins , 39-51.

Lande, R., 1987. Extinction thresholds in demographic models of territorial populations. The American Naturalist 130, 624-635.

Lande, R., 1993. Risks of population extinction from demographic and environmental stochasticity and random catastrophes. The American Naturalist 142, 911-927.

Lande, R., Engen, S., Sæther, B.E., 2009. An evolutionary maximum principle for density-dependent population dynamics in a fluctuating environment. Philosophical Transactions of the Royal Society B: Biological Sciences 364, 1511-1518.

Lele, S.R., Dennis, B., Lutscher, F., 2007. Data cloning: easy maximum likelihood estimation for complex ecological models using bayesian markov chain monte carlo methods. Ecology letters 10, 551-563.

Li, T.Y., Yorke, J.A., 2004. Period three implies chaos, in: The Theory of Chaotic Attractors. Springer, pp. $77-84$

Loeschcke, V., Seitz, A., 1991. Species conservation: a population-biological approach. Citeseer.

Luckinbill, L.S., 1973. Coexistence in laboratory populations of paramecium aurelia and its predator didinium nasutum. Ecology 54, 1320-1327.

Ludwig, D., 1999. Is it meaningful to estimate a probability of extinction? Ecology 80, 298-310.

MacArthur, R.H., 1962. Some generalized theorems of natural selection. Proceedings of the National Academy of Sciences of the United States of America 48, 1893.

McCarthy, M.A., Possingham, H.P., Day, J.R., Tyre, A., 2001. Testing the accuracy of population viability analysis. Conservation Biology 15, 1030-1038.

Méndez, V., Llopis, I., Campos, D., Horsthemke, W., 2010. Extinction conditions for isolated populations affected by environmental stochasticity. Theoretical population biology 77, 250-256.

Mortoja, S.G., Panja, P., Paul, A., Bhattacharya, S., Mondal, S.K., 2020. Is the intermediate predator a key regulator of a tri-trophic food chain model?: An illustration through a new functional response. Chaos, Solitons \& Fractals 132, 109613.

Münzbergová, Z., Ehrlén, J., 2005. How best to collect demographic data for population viability analysis models. Journal of applied Ecology 42, 1115-1120.

Nicholson, A.J., 1954. An outline of the dynamics of animal populations. Australian journal of Zoology 2, 9-65. 
Nicolau, J., 2002. A new technique for simulating the likelihood of stochastic differential equations. The Econometrics Journal 5, 91-103.

Nicolau, J., 2004. Introduction to the estimation of stochastic differential equations based on discrete observations, in: Autumn School and International Conference, Stochastic Finance.

Ozaki, T., 1992. A bridge between nonlinear time series models and nonlinear stochastic dynamical systems: a local linearization approach. Statistica Sinica , 113-135.

Pe'er, G., Matsinos, Y.G., Johst, K., Franz, K.W., Turlure, C., Radchuk, V., Malinowska, A.H., Curtis, J.M., NAUJOKAITIS-LEWIS, I., Wintle, B.A., et al., 2013. A protocol for better design, application, and communication of population viability analyses. Conservation Biology 27, 644-656.

Pianka, E.R., 1970. On r-and k-selection. The american naturalist 104, 592-597.

NERC Centre for Population Biology, I.C., 2010. The global population dynamics database.

R Core Team, 2019. R: A Language and Environment for Statistical Computing. R Foundation for Statistical Computing. Vienna, Austria. URL: https://www.R-project.org/.

Rana, S., Bhowmick, A.R., Bhattacharya, S., 2014. Impact of prey refuge on a discrete time predatorprey system with allee effect. International Journal of Bifurcation and Chaos 24, 1450106.

Reed, J.M., Murphy, D.D., Brussard, P.F., 1998. Efficacy of population viability analysis. Wildlife Society Bulletin , 244-251.

Reynolds, J.D., Freckleton, R.P., 2005. Population dynamics: growing to extremes. Science 309, 567-568.

Ritchie, M.E., 1992. Chaotic dynamics in food-limited populations: implications for wildlife management, in: Wildlife 2001: populations. Springer, pp. 139-147.

Rosenzweig, M.L., 1971. Paradox of enrichment: destabilization of exploitation ecosystems in ecological time. Science 171, 385-387.

Ross, J., 2009. A note on density dependence in population models. Ecological Modelling 220, 3472-3474.

Roughgarden, J., 1979. Theory of population genetics and evolutionary ecology: an introduction.

Ruxton, G.D., 1994. Low levels of immigration between chaotic populations can reduce system extinctions by inducing asynchronous regular cycles. Proceedings of the Royal Society of London. Series B: Biological Sciences 256, 189-193.

Sæther, B.E., Engen, S., Islam, A., McCleery, R., Perrins, C., 1998. Environmental stochasticity and extinction risk in a population of a small songbird, the great tit. The American Naturalist 151, $441-450$.

Saha, B., Bhowmick, A.R., Chattopadhyay, J., Bhattacharya, S., 2013. On the evidence of an allee effect in herring populations and consequences for population survival: A model-based study. Ecological modelling 250, 72-80.

Sau, A., Saha, B., Bhattacharya, S., 2020. An extended stochastic allee model with harvesting and the risk of extinction of the herring population. Journal of Theoretical Biology 503, 110375. 
Schiegg, K., Walters, J.R., Priddy, J.A., 2005. Testing a spatially explicit, individual-based model of red-cockaded woodpecker population dynamics. Ecological Applications 15, 1495-1503.

Schreiber, S.J., 2003. Allee effects, extinctions, and chaotic transients in simple population models. Theoretical population biology 64, 201-209.

Shaffer, M.L., 1981. Minimum population sizes for species conservation. BioScience 31, 131-134.

Shoji, I., Ozaki, T., 1998. Estimation for nonlinear stochastic differential equations by a local linearization method. Stochastic Analysis and Applications 16, 733-752.

Sibly, R.M., Barker, D., Denham, M.C., Hone, J., Pagel, M., 2005. On the regulation of populations of mammals, birds, fish, and insects. Science 309, 607-610.

Sibly, R.M., Barker, D., Hone, J., Pagel, M., 2007. On the stability of populations of mammals, birds, fish and insects. Ecology Letters 10, 970-976.

Sjögren-Gulve, P., Hanski, I., 2000. Metapopulation viability analysis using occupancy models. Ecological bulletins , 53-71.

Taylor, B.L., 1995. The reliability of using population viability analysis for risk classification of species. Conservation Biology 9, 551-558.

Thomas, W.R., Pomerantz, M.J., Gilpin, M.E., 1980. Chaos, asymmetric growth and group selection for dynamical stability. Ecology 61, 1312-1320.

Thunberg, H., 2001. Periodicity versus chaos in one-dimensional dynamics. SIAM review 43, 3-30.

Uchida, M., Yoshida, N., 2005. Aic for ergodic diffusion processes from discrete observations. preprint MHF 12.

Veilleux, B., 1979. An analysis of the predatory interaction between paramecium and didinium. The Journal of Animal Ecology , 787-803.

Waliszewski, P., Konarski, J., 2005. A mystery of the gompertz function, in: Fractals in biology and medicine. Springer, pp. 277-286.

Welch, P., Benner, K., Leaf, B., 2007. Calibration of assessment methods for fraser river sockeye salmon (oncorhynchus nerka) spawning populations $(25,000$ to 75,000$)$ in the horsefly, stellako and adams river systems. canada department of fisheries and oceans, vancouver. British Columbia .

Yoshida, N., 1992. Estimation for diffusion processes from discrete observation. Journal of Multivariate Analysis 41, 220-242. 


\section{Supplementary Files}

This is a list of supplementary files associated with this preprint. Click to download.

- AyanSupplementary1.pdf

- AyanSupplementary2.xlsx 